\title{
Quantization of Planetary Systems and its Dependency on Stellar Rotation
}

\author{
Jean-Paul A. Zoghbi \\ American University of Beirut, Bliss St., Beirut, Lebanon. \\ Corresponding author. Email: jeanpaulis@yahoo.com \\ Received 2009 September 24, accepted 2011 February 28
}

\begin{abstract}
With the discovery of now more than 500 exoplanets, we present a statistical analysis of the planetary orbital periods and their relationship to the rotation periods of their parent stars. We test whether the structural variables of planetary orbits, i.e. planetary angular momentum and orbital period, are 'quantized' in integer or half-integer multiples of the parent star's rotation period. The Solar System is first shown to exhibit quantized planetary orbits that correlate with the Sun's rotation period. The analysis is then expanded over 443 exoplanets to statistically validate this quantization and its association with stellar rotation. The results imply that the exoplanetary orbital periods are highly correlated with the parent star's rotation periods and follow a discrete half-integer relationship with orbital ranks $n=0.5,1.0,1.5,2.0,2.5$, etc. The probability of obtaining these results by pure chance is $p<0.024$. We discuss various mechanisms that could justify this planetary quantization, such as the hybrid gravitational instability models of planet formation, along with possible physical mechanisms such as the inner disc's magnetospheric truncation, tidal dissipation, and resonance trapping. In conclusion, we statistically demonstrate that a quantized orbital structure should emerge from the formation processes of planetary systems and that this orbital quantization is highly dependent on the parent star's rotation period.
\end{abstract}

Keywords: planetary systems: formation — star: rotation — solar system: formation

\section{Introduction}

The discovery of now more than 500 exoplanets has provided the opportunity to study the various properties of planetary systems and has considerably advanced our understanding of planetary formation processes. One long-suspected property of planetary systems has been the quantum-like feature that resembles the mathematical regularity of the empirical Titius-Bode (TB) law in the Solar System. Various research papers have suggested that such 'quantized' features and empirical relationships might be possible in extra-solar multi-planetary systems, such as Nottale (1996; 1997b), Nottale et al. (1997a; 2004), Rubcic \& Rubcic (1998; 1999), Poveda \& Lara (2008), and Chang (2010), just to mention a few. In case they truly exist, one main question that needs to be answered is what physical processes might cause these 'quantization' features to develop. The gravitational instability model of planet formation has been successfully used in the past to explain 'discrete' power law distributions in planetary spacing (Griv \& Gedalin 2005). Similarly, hybrid models of planetary formation (e.g. Durisen et al. 2005), are characterised by concentric dense gas rings that are produced by resonances and discrete spiral modes which, in theory, can be correlated with orbital 'quantization' features. Also similarly, tidal dissipation and angular momentum transfer, along with mean-motion resonances and resonance trapping, play an important role in the final orbital configuration. In all of these mechanisms, the stellar rotation period is a critical parameter. The main motivation in this paper is therefore (i) to statistically search for any apparent quantum-like features in the orbital structure of exoplanetary systems; (ii) to determine whether the quantization parameters are related to any specific physical system property (the stellar rotation rate is examined in this paper); and (iii) to shed some light on the nature of the possible physical processes that might lead to this apparent quantization. We will argue on dynamical terms that a quasi-quantum model might emerge naturally from the formation processes that determine the final configuration of a planetary system.

The plan of this paper is as follows: Section 2 describes the basic methodology and simple quantum-like model. In Section 3, the model is applied to the Solar System. In Section 4, the analysis is expanded over a sample of 443 exoplanets, for which we could obtain stellar rotation periods. In Section 5, a statistical analysis of the results is presented demonstrating that the specific angular momenta of all planetary orbits generally follow half-integer multiples of the specific angular momentum at the parent star's corotation radius. Section 6 briefly proposes various physical mechanisms that may justify the obtained results. Prospects and conclusions are drawn in Section 7. 


\section{Methodology}

We will be testing for the quantization of planetary angular momentum, i.e. we will test whether planetary angular momenta have discrete values in multiples of a 'ground-state' system-specific parameter. Within an order of magnitude estimate, the corotation orbit represents an approximate inferior limit to the position of planetary orbits. This is confirmed by various physical mechanisms that are discussed in Section 6, such as spin-orbit coupling and tidal dissipation, as well as disc-locking and magnetic braking which create a barrier and inferior limit to planetary migration. On that basis, we postulate that the corotation orbit represents the ground-state orbit of planetary systems and assign to it the orbital rank $n=1$. However, this does not negate the possibility of having physical objects orbiting inside the corotation orbit. Nevertheless, the corotation orbit (at $n=1$ ) is particularly chosen because of its importance as a base reference to the orbital parameters of the entire planetary system, and in particular, their relationship to the parent star's rotation period.

The corotation radius $r_{0}$ is defined in terms of the star's rotation rate $\Omega_{\mathrm{s}}$ by

$$
r_{0}=\left(\frac{G M}{\Omega_{s}^{2}}\right)^{1 / 3},
$$

where $G$ is the gravitational constant and $M$ and $\Omega_{\mathrm{s}}$ are the mass and rotation rate of the parent star respectively. Similarly, the mean motion orbital velocity $v_{0}$ and specific angular momentum $J_{0}$ (per unit mass) at the corotation radius are given by

$$
\begin{aligned}
& v_{0}=\left(\frac{G M}{r_{0}}\right)^{1 / 2}=\left(G M \Omega_{s}\right)^{1 / 3}, \\
& J_{0}=v_{0} r_{0}=\left(\frac{G^{2} M^{2}}{\Omega_{s}}\right)^{1 / 3}
\end{aligned}
$$

If our planetary quantization hypothesis is valid, then the specific angular momentum $J_{\mathrm{n}}$ of any planetary orbit $n$ would follow discrete and quantized multiples of the specific angular momentum $J_{0}$ at the corotation orbit $n=1$.

$$
J_{n}=n J_{0}=n\left(\frac{G^{2} M^{2}}{\Omega_{s}}\right)^{1 / 3}
$$

In other words, the ratio of the specific orbital angular momentum $J_{\mathrm{n}}$ of a planet in the $n^{\text {th }}$ planetary orbit to the specific angular momentum $J_{0}$ at the 'ground-state' corotation orbit ought to increase incrementally by a discrete value.

$$
n=\frac{J_{n}}{J_{0}}
$$

For nearly circular orbits, Newton's force-balance equation of motion gives

$$
\begin{gathered}
r_{n}=\frac{G M}{v_{n}{ }^{2}}, \text { and } \\
J_{n}=v_{n} r_{n}=\left(G M r_{n}\right)^{1 / 2}
\end{gathered}
$$

Combining equations (1), (5), and (7), we obtain an $n^{2}$ law for the quantized semi-major axis $r_{n}$ of the $n^{\text {th }}$ orbit, in terms of the corotation radius $r_{0}$, spin rotation rate $\Omega_{\mathrm{s}}$ of the central star, and orbital rank $n$ by

$$
r_{n}=n^{2} r_{0}=n^{2}\left[\frac{G M}{\Omega_{s}^{2}}\right]^{1 / 3}
$$

From Kepler's third law and Equation (8), the quantized orbital period $P_{n}$ of the $n^{\text {th }}$ planetary orbit is also given in terms of the corotation orbital period $P_{0}$ and orbital rank $n$ by

$$
\mathrm{P}_{n}=n^{3} \mathrm{P}_{0}=n^{3} \mathrm{P}_{\text {rot }}, \text { or } n=\left(\frac{\mathrm{P}_{n}}{\mathrm{P}_{\text {rot }}}\right)^{1 / 3}
$$

Where $P_{\mathrm{n}}$ is the planet's orbital period and $P_{0}$ is the corotation period which is by definition equal to $P_{\text {rot }}$, the star's rotation period.

\section{Solar System Application and Results}

\subsection{The Solar System Orbital Ranks}

The quantum-like model described in Section 2 is first applied to the planets of the Solar System in order to discern any discrete pattern in their orbital ranks $n$. We will calculate the orbital ranks $n$ using the Sun's rotation period $P_{\text {Sun }}$ taken from Allan's Astrophysical Quantities (Cox 1999) as 25.38 days and the solar rotation rate $\Omega_{\text {Sun }}=2.8 \times 10^{-6} \mathrm{rd} \mathrm{s}^{-1}$. The Sun's corotation specific angular momentum $J_{0}$ is calculated from Equation (3) and will represent the base quantization parameter for all possible planetary orbits in the Solar System. The planetary orbital ranks are first calculated using the Sun's present rotation period (25.38 days) and presented in Table 1. However, since the Sun's rotation rate has already decayed with age through angular momentum loss, the orbital ranks are also calculated using the Sun's rotation rate at the early stage of planet formation. The orbital parameters of the Solar System are assumed to have settled into a long-term stable configuration at around $650 \mathrm{Myr}$ or so. Using data on solar-type stars in the Hyades (age $\sim 650 \mathrm{Myr}$ ), we selected star VB-15 which has a $B-V$ index similar to the Sun's to estimate the Sun's rotation period of 8 days (Radick et al. 1987) at the planets' formation age, i.e. the time when the solar system planets' orbits have stabilised and the proposed quantization has 'frozen in'. The planets' orbital ranks $n$ are calculated from Equation (5) for each planetary orbit using both the Sun's present and earlier formation rotation 


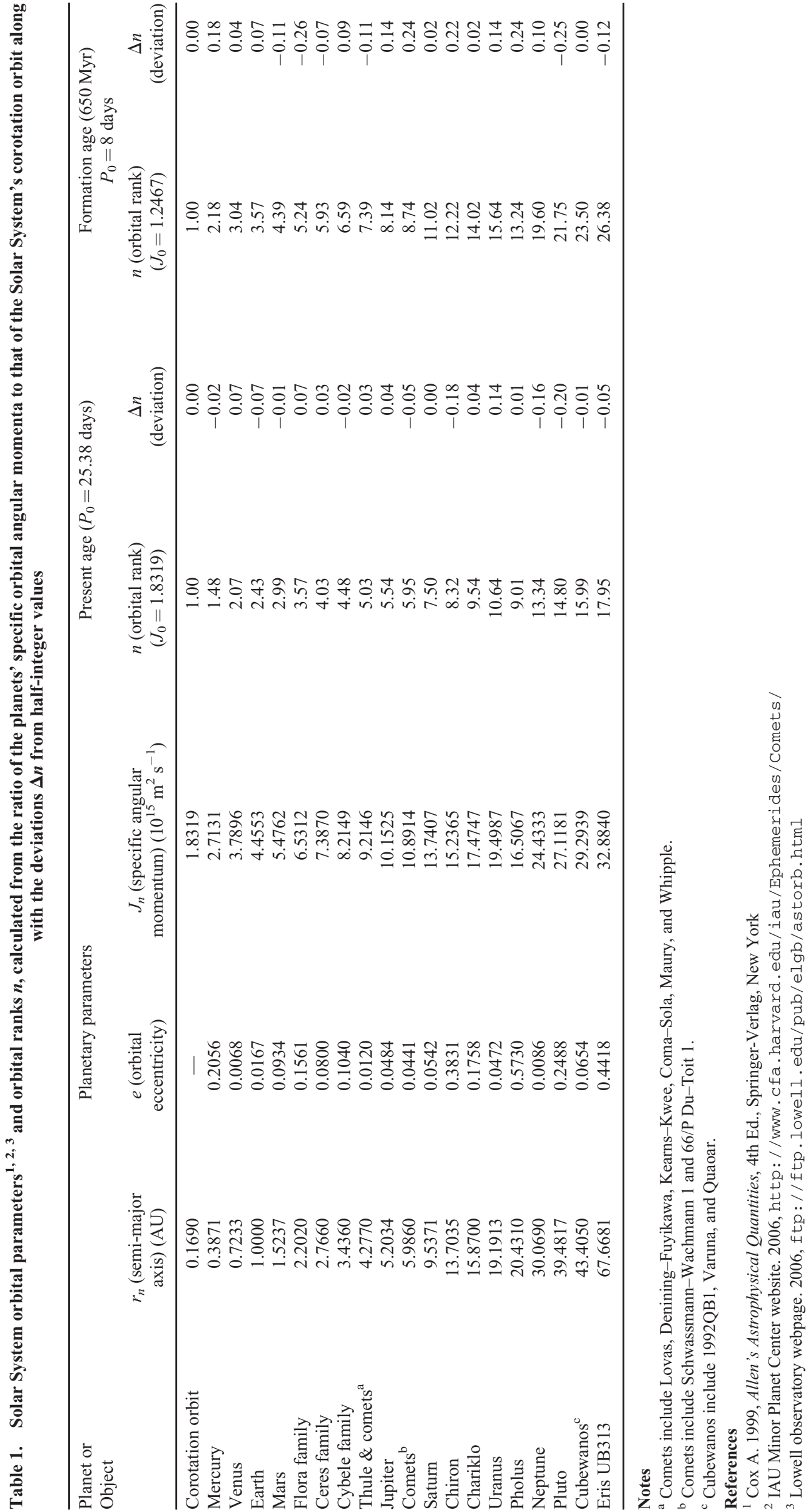


periods $\left(J_{0}=1.8319\right.$ and $1.2467 \mathrm{~m}^{2} \mathrm{~s}^{-1}$ respectively). The results are presented in Table 1 .

Besides the main planets, the list includes main massdistribution peaks such as the asteroid belt families: Flora, Ceres, Pallas, Cybele, and Thule, as well as centaurs, trans-Neptunian cubewanos in the Kuiper belt, and the recently discovered scattered disc object (SDO) 2003UB313, previously dubbed the 'tenth planet'.

From Table 1, it can be observed from the orbital ranks calculated using the Sun's present rotation period that the Solar System exhibits a discrete and quantized orbital structure where the planets' specific orbital angular momenta $J_{n}$ are ranked in discrete half-integer multiples of the specific angular momentum $J_{0}$ at the solar corotation orbit $(n=1.0,1.5,2.0,2.5,3.0,3.5$, etc.). The $\Delta n$ deviations from integer or half-integer values are included in Table 1 and indicate that 16 out of 19 planetary orbits have absolute deviations $|\Delta n|<0.07$. The discrete nature of planetary semi-major axes, mean orbital velocities, and orbital periods, in terms of half-integer values, follows logically from the quantized orbital angular momentum results.

The inner planets Mercury $(n=1.5)$, Venus $(n=2.0)$, Earth $(n=2.5)$, and Mars $(n=3.0)$ occupy the ranks $n=1.48,2.07,2.43$, and 2.99 respectively with minimal deviations $\Delta n$ from the closest integer or half-integer values. In the main asteroid belt, the orbits of the Flora family are ranked at $n=3.5$, with both Flora and Ariadne occupying $n=3.57$. At the orbital rank $n=4$, the main asteroid families of Ceres and Pallas represent the group and both occupy the rank $n=4.03$. This orbital rank also includes Misa, Eunomia, Lamberta, and the Chloris families at $n=3.90$; Ino and Adeana at $n=3.94$; Dora at $n=3.96$; Elpis, Herculina, Gyptis, Juewa, Minerva, Thisbe, Dynamene, and Eunike, all at $n=3.99$; Eugenia and Nemesis at $n=4.0$; Lydia, Gefion, and Pompeja at $n=4.01$; and the Brasilia and Karin families at $n=4.09$.

The orbits of the Cybele family of asteroids are ranked at $n=4.5$, with the main asteroid Cybele, for instance, occupying rank $n=4.48$, Sibylla and Hermione at $n=$ 4.47, Bertholda at $n=4.49$, Camilla at $n=4.52$, and Sylvia at $n=4.53$. At the next orbital rank of $n=5$, the main asteroid Thule occupies $n=5.03$. At the orbital rank of $n=5.5$, Jupiter occupies the rank $n=5.54$ along with the Trojan asteroids such as Achilles at $n=5.48$, Diomedes at $n=5.49$, Aeneas at $n=5.50$, Patroclus and Nestor at $n=5.51$, Hektor at $n=5.53$, and Agamemnon at $n=5.54$. Beyond Jupiter, the orbital ranks at $n=6.0$, $n=6.5$, and $n=7.0$ do not appear to be occupied by any major object. However, this does not exclude various periodic comets whose orbital properties match several orbital ranks in the Solar System. To mention a few, the comets 29/P Schwassmann-Wachmann-1 and 66/P Du-Toit both occupy $n=5.95$ and $n=5.94$ respectively. Ranked also with the asteroid Thule, for instance, are the comets 36/P Whipple at $n=4.97,115 / \mathrm{P}$ Maury at $n=5.01,32 / \mathrm{P}$ Coma-Sola at $n=5.02,59 / \mathrm{P}$ Kearns-Kwee at $n=5.05,72 / \mathrm{P}$ Denning-Fuyikawa at $n=5.06$, and 93/P
Lovas at $n=5.08$. However, the unoccupied ranks beyond Jupiter are better explained by orbital migration and the outward expansion of the Solar System's boundaries.

Saturn occupies the rank $n=7.50$ exactly, followed by 'centaurs' such as Chiron at $n=9.01$, Chariklo at $n=$ 9.69, and Pholus at $n=10.97$. Uranus occupies the rank $n=10.65$ and Neptune $n=13.34$, with relatively higher but nearly equal and opposite deviations from integer or half-integer ranking, $\Delta n=+0.14$ and -0.16 respectively. At the orbital rank of $n=15.5$, Pluto occupies $n=15.28$. Beyond Pluto and at the orbital rank $n=16$, some cubewanos, classified as trans-Neptunian objects (TNO), in the Kuiper belt are included, such as Quaoar at $n=16.00$ and Varuna at $n=15.97$. The recently discovered SDO Eris UB313 occupies the rank $n=17.95$. This can be used to predict the location of objects within and beyond the SDOs. At $n=20$, for instance, an object may be discovered orbiting at $67.85 \mathrm{AU}$.

We note that the deviations $\Delta n$ from the closest integer or half-integer are negligible up to Saturn and all orbital ranks are occupied by planets or asteroid mass peaks within that region. Beyond Saturn's orbit, the deviations $\Delta n$ are relatively higher for Uranus, Neptune and Pluto and several orbital ranks are vacant. One possible explanation may be related to dissipation in the solar protoplanetary disc that allows both inward and outward planetary migrations, depending on the initial position and the radius of maximum viscous stress located just outside the orbit of Saturn (at around 10 AU). Hence, the orbits of protoplanets forming within that critical radius tend to compact, while those forming outside it are stretched outwards. This could explain the relatively higher $\Delta n$ deviations beyond Saturn's orbit and, more importantly, the unoccupied orbital ranks produced by the outward expansion.

The orbital ranks $n$ that were calculated using the Sun's rotation period (8 days) at the formation age of $650 \mathrm{Myr}$ also exhibit a discrete and quantized structure, albeit with higher deviations $\Delta n$ from half-integer numbers. It can therefore be inferred that the decay in solar rotation rate has improved or at least had a minimal effect on the quantized orbital ranks, most likely because the orbital ranks are proportional to the cube root of the decreasing rotation rate (Equation 5). The slowing down of the Sun's rotation rate has actually improved the discrete quantized nature of the orbital structure with deviations from halfintegers approaching zero as the rotation rate decreases asymptotically with age (Skumanich 1972), where it reaches a limit value that has negligible further effect to the orbital rank values. This effect is more clearly seen in Section 4.1, where the model is applied to 443 exoplanets.

\section{Exoplanetary Application and Results}

\subsection{Exoplanetary Orbital Ranks at Parent Stars' Present Rotation Periods}

To date, more than 500 exoplanets with 49 multiplanetary systems have been discovered. In order to verify 
Table 2. Rotation periods of Hyades stars used to match the $B-V$ colour of exoplanetary stars

\begin{tabular}{|c|c|c|c|c|c|c|c|c|c|}
\hline $\begin{array}{l}\text { VB } \\
\text { No. }\end{array}$ & $B-V$ & $\begin{array}{l}\text { Represents } \\
B-V \text { range }\end{array}$ & $\begin{array}{c}P_{\text {rot }} \\
\text { (days) }\end{array}$ & Ref. & $\begin{array}{l}\text { VB } \\
\text { No. }\end{array}$ & $B-V$ & $\begin{array}{l}\text { Represents } \\
B-V \text { range }\end{array}$ & $\begin{array}{c}P_{\text {rot }} \\
\text { (days) }\end{array}$ & Ref. \\
\hline VB 94 & 0.396 & Less than 0.430 & 1.67 & $(2)$ & VB 17 & 0.706 & 0.690 to 0.710 & 7.25 & (2) \\
\hline VB 78 & 0.451 & 0.431 to 0.460 & 2.90 & (1) & VB 27 & 0.721 & 0.711 to 0.730 & 7.15 & (2) \\
\hline VB 81 & 0.470 & 0.460 to 0.480 & 2.80 & (1) & VB 92 & 0.736 & 0.731 to 0.740 & 9.13 & (6) \\
\hline VB 121 & 0.500 & 0.480 to 0.510 & 3.70 & (7) & VB 26 & 0.745 & 0.741 to 0.760 & 9.06 & (5) \\
\hline VB 48 & 0.518 & 0.511 to 0.520 & 2.50 & (1) & VB 22 & 0.770 & 0.761 to 0.780 & 5.61 & (7) \\
\hline VB 65 & 0.535 & 0.521 to 0.530 & 5.87 & $(5)$ & VB 3 & 0.786 & 0.781 to 0.799 & 12.04 & (2) \\
\hline VB 59 & 0.543 & 0.531 to 0.545 & 5.13 & $(5)$ & VB 21 & 0.816 & 0.800 to 0.819 & 5.49 & (3) \\
\hline VB 29 & 0.548 & 0.546 to 0.559 & 3.00 & (1) & VB 79 & 0.831 & 0.820 to 0.839 & 9.71 & (6) \\
\hline VB 119 & 0.563 & 0.560 to 0.569 & 4.00 & (7) & VB 153 & 0.855 & 0.840 to 0.869 & 9.18 & (4) \\
\hline VB 31 & 0.572 & 0.570 to 0.579 & 4.72 & (3) & VB 138 & 0.871 & 0.870 to 0.879 & 19.19 & (2) \\
\hline VB 52 & 0.592 & 0.580 to 0.599 & 5.64 & (3) & VB 43 & 0.907 & 0.880 to 0.920 & 10.26 & (5) \\
\hline VB 50 & 0.604 & 0.600 to 0.609 & 5.10 & (1) & VB 91 & 0.936 & 0.921 to 0.970 & 9.36 & (5) \\
\hline VB 73 & 0.609 & 0.610 to 0.620 & 7.38 & (6) & VB 25 & 0.984 & 0.971 to 1.010 & 12.64 & (5) \\
\hline VB 97 & 0.624 & 0.621 to 0.629 & 6.45 & (3) & VB 175 & 1.031 & 1.011 to 1.080 & 10.82 & (5) \\
\hline VB 18 & 0.640 & 0.630 to 0.649 & 8.65 & (4) & VB 181 & 1.167 & 1.081 to 1.199 & 11.92 & (5) \\
\hline VB 63 & 0.651 & 0.650 to 0.653 & 7.73 & $(5)$ & VB 173 & 1.237 & 1.200 to 1.300 & 14.14 & (5) \\
\hline VB 15 & 0.657 & 0.654 to 0.660 & 7.43 & (3) & VB 190 & 1.357 & 1.301 to 1.499 & 3.66 & (5) \\
\hline VB 64 & 0.664 & 0.661 to 0.670 & 8.64 & $(6)$ & $\mathrm{H} 218$ & M3 M4 & 1.511 to 1.600 & 0.68 & (8) \\
\hline VB 58 & 0.680 & 0.671 to 0.689 & 6.20 & (1) & & & & & \\
\hline
\end{tabular}

\section{References}

(1) Duncan et al. 1984, (2) Glebocki \& Gnacinski 2005, (3) Paulsen et al. 2003, (4) Paulsen et al. 2004, (5) Radick et al. 1987, (6) Radick et al. 1995, (7) Rutten 1987, (8) Scholz \& Eisloffel 2007

whether the quantization of planetary angular momentum in discrete half-integer values is a universal occurrence and not just a coincidental property of the Solar System, and in order to validate the dependency of this quantization on stellar rotation, a sample of 443 exoplanets, for which star rotation data is available, is analysed with respect to the rotation periods of their parent stars. Out of the 443 exoplanets, almost half (216 stars) have host stars with rotation periods available from literature or measured from $\log R_{H K}^{\prime}$. These were obtained from the planets' discovery papers (49 parent stars; see references in Table 3) or from Table 1 of Watson et al. (2010) (167 parent stars) which conveniently compiles all published rotation periods of exoplanetary host stars. We preferred to use Table 1 of Watson et al. (2010) and not Tables 2 and 3 , as Table 1 compiles published rotation periods while Table 2 and 3 use Markov-chain Monte Carlo simulation to estimate them. Out of the 167 rotation periods in Watson et al. (2010), 7 stars have actual observed rotation period and these are: Rho $\mathrm{CrB}$ (17 days), Tau Boo (14 days), Epsilon Eri (11.68 days), HD 3651 (44 days), HD 62509 (135 days), HD 70573 (3.3 days), HD 89744 (9 days). The remaining 227 stellar rotation periods were estimated from the projected rotational velocities $v \sin i$ and stellar radii, with certain levels of uncertainty. We have used the Catalogue of Nearby Exoplanets (Butler et al. 2006), the Catalogue of Rotational Velocities (Glebocki \& Gnacinski 2005), the Exoplanet Data Explorer Table (http : / / exoplanets . org/; Wright \& Marcy 2010), along with some planet discovery papers, to obtain values of $v \sin i$ (see references in Table 3). We noted that in many cases the values of $v \sin i$ listed in the Exoplanet Data Explorer Table were truncated and rounded up, which is why we attempted as much as possible to obtain more accurate values (to 2 significant digits) from the referenced papers. Moreover, in some cases where the catalogues listed multiple values of $v \sin i$ for a particular star, the values listed as upper limits were generally avoided and similar values when measured and corroborated by different sources were selected. The absolute stellar radii were mainly taken from the referenced planet discovery papers, the Fundamental Parameters of Stars Catalogue (Allende, Prieto \& Lambert 1999), the Catalogue of Stellar Diameters (Pasinetti Fracassini et al. 2001), or the Effective Temperatures and Radii of Stars Catalogue (Masana et al. 2006).

\subsection{Uncertainty Considerations}

Most of the $v \sin i$ values we found are under $4 \mathrm{~km} \mathrm{~s}^{-1}$. The inherent measurement uncertainty in these $v \sin i$ values is at best around $0.5 \mathrm{~km} \mathrm{~s}^{-1}$ and at worst 1 to $2 \mathrm{~km} \mathrm{~s}^{-1}$. As for stellar radii, although they can be determined to a precision on the order of 5 percent for the small minority of planets that transit their parent stars, the radius estimates for the remainder are unlikely to be established to a precision better than 10 percent. Moreover, the orbital angular momenta of exoplanets depend on stellar mass values which are mostly derived from isochrone fits and have an inherent uncertainty on the order of 10 percent. Therefore, the extra-solar orbital ranks, if calculated from the planetary angular momenta in Equation (5), would be uncertain by at least 30 percent (since $M_{\mathrm{S}}$ and $R_{\mathrm{s}}$ are correlated on the main sequence) even before the uncertainties in the measured $v \sin i$ (a further 30 percent) and the effects of unknown orbital inclination are taken into account. 
Fortunately the dependence on stellar mass in Equation (5) can be eliminated. Equation (9) is the key, as it uses the orbital period of the planet and rotation period of the star directly. By using Equation (9) to calculate the orbital ranks, the use of the planet's semi-major axis is avoided, as it requires knowledge of the uncertain stellar mass. Since the orbital period is one of the few planetary parameters that is measured directly and with high precision, this is the best quantity to use. The same is true for almost half of the exoplanet sample having directly measured stellar rotation periods. The remaining rotation periods were derived from measurements of $v \sin i$, which are typically uncertain by 20 to 30 percent, and estimates of stellar radii are also uncertain by roughly 10 percent. This may not be disastrous, since the ratio of the two values is raised to the one-third power. Therefore, the final estimates for the orbital ranks $n$ are expected to be uncertain by 5 to 10 percent. Moreover, the uncertainty due the unknown inclination of the stellar rotation axis takes the form of $(\sin i)^{-1 / 3}$. For inclination angles ranging from $45^{\circ}$ to $90^{\circ}$, this factor is very close to unity, and therefore has an insignificant effect on the calculated orbital ranks $n$. For inclinations between $30^{\circ}$ and $45^{\circ}$, the $(\sin i)^{-1 / 3}$ factor can affect the $n$ values by as much as 10 to 20 per cent. However, since the most likely inclination of a random stellar sample is $57^{\circ}$ (Trilling et al. 2002) and because the radial velocity technique is biased towards detecting planetary systems with inclinations near $90^{\circ}$, the average value of is expected to range between $\pi / 4$ and unity. With the number of exoplanets under consideration, the average value of $\sin i$ for the population approaches the value for a random distribution. Hence, the most likely effect of the inclination factor on the calculated orbital ranks should again not exceed 7 to 10 per cent on average. A Monte Carlo treatment is used in Section 5 to study the effect of these uncertainties.

In the first approach, the extra-solar orbital ranks $n$ and their related deviations from half-integer values $\Delta n$ are calculated using the ratio of the planet's orbital period to that of the parent star's current rotation period, as in Equation (9), and presented in Table 3.

\subsection{Exoplanetary Orbital Ranks at the Planetary Formation Epoch ( 650 Myr)}

In the second approach, we address the concern that the half-integer orbital ranks are calculated using the rotation period for the present age of the star, and not at the epoch when planetary systems were formed, when it is known that solar-type stars observed in young star clusters do not rotate at constant rates throughout their lifetimes. We therefore need to study whether any quantization feature exist at the formation age when it is supposedly 'frozen in'.

The rotation rates of stars with outer convection zones generally decay with age, approximately as the inverse square root of time (Skumanich 1972), through angular momentum loss via hot magnetically channeled winds. However, at around $600 \mathrm{Myr}$ or so, planetary systems eventually settle into long-term stable configurations and their orbital periods are constant while the stellar rotation periods continue to increase. However, Soderblom et al. (2001) indicate that the rotation of solar-type stars, in evolving from the Pleiades (100 Myr) to the Hyades (650 Myr), changes only modestly in the mean, but undergoes a huge convergence in the spread of rotation rates. Thus, at any one mass in the Pleiades (100 Myr), the range of rotation rates varies by an order of magnitude or more, yet in the Hyades (650 Myr), stars of the same mass have nearly identical rotation rates. The convergence occurs for upper bound rotation rates, as the lower bounds of both clusters are nearly identical (Soderblom et al. 2001). Since most of our sample of exoplanetary stars have rotation periods near the lower bounds, we can assume that these have remained essentially unchanged over the period 100-650 Myr (Soderblom et al. 2001), i.e. during the period of planetary formation. With this minimal decay in rotation periods, it is therefore logical to expect a quantized distribution of orbital ranks around half-integer values at the early formation age period.

Nevertheless, rather than using only the present rotation periods of these stars, we additionally adopt a stellar rotation period at the fiducial planet-formation age (650 Myr), that can be derived from the star's $B-V$ colour and the known rotation periods of stars of the same colour in the Hyades (aged 650 Myr). This procedure has the advantage that it can be based on direct measurements of stellar rotation periods in stellar clusters of known age, near the epoch when the proposed planetary quantization would have been established. This bypasses the difficulties arising from the uncertainties in $v \sin i$, inclination, stellar radius, and stellar age.

Table 2 presents the Hyades stars, their $B-V$ color, and directly measured rotation periods which were used in matching the 443 exoplanetary star sample. The Hyades stars' $B-V$ colour range from 0.41 to 1.53 . However, we could not find any measured rotation periods for Hyades stars in the $B-V$ ranges from 0.30 to $0.40,0.69$ to 0.73 , 0.78 to 0.80 , and 0.87 to 0.88 . Instead, five Hyades stars (HD 28911, HD 26756, HD 27282, HD 21663, and HD 26397 ) with rotation periods derived from $v \sin i$ and stellar radii were selected to supplement for the missing $B-V$ ranges. Additionally, one Praesepe star H218 (of similar age $\sim 650 \mathrm{Myr}$ ), having a directly measured rotational period, was selected to cover for the few exoplanetary stars of similar spectral type, M2 to M4 (Scholz \& Eisloffel 2007). A Monte Carlo treatment is presented in Section 5 to address the inherent uncertainty in these rotation periods.

The $B-V$ colour values for the exoplanetary stars were obtained primarily from the All-sky Compiled Catalogue of 2.5 Million Stars (Kharchenko 2001), the NOMAD Catalog (Zacharias et al. 2005), and the Hipparcos \& Tycho Catalogue (ESA 1997) and were matched with the corresponding value from the Hyades stars to obtain an estimate of their early rotation periods at the age of $650 \mathrm{Myr}$. These rotation periods were then used to 


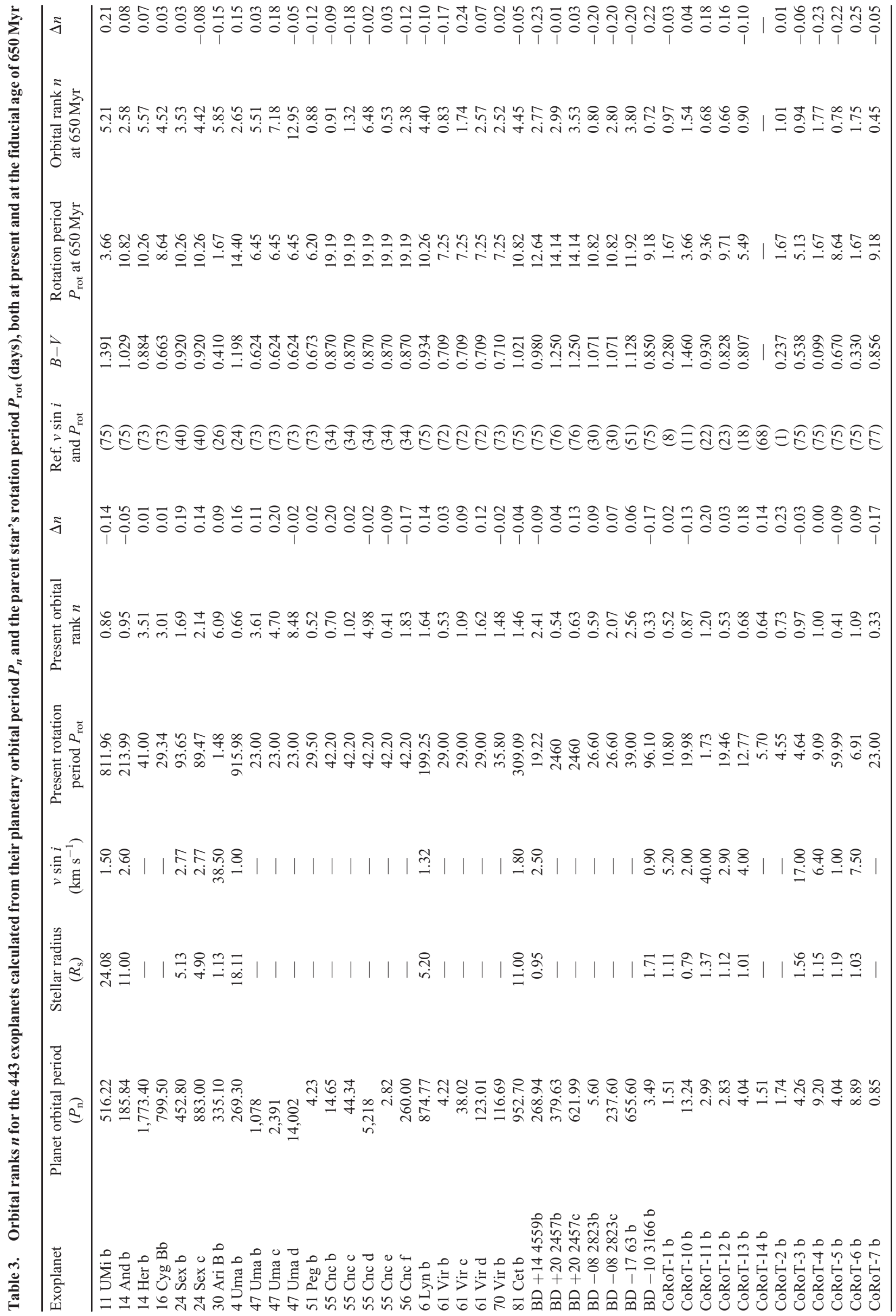




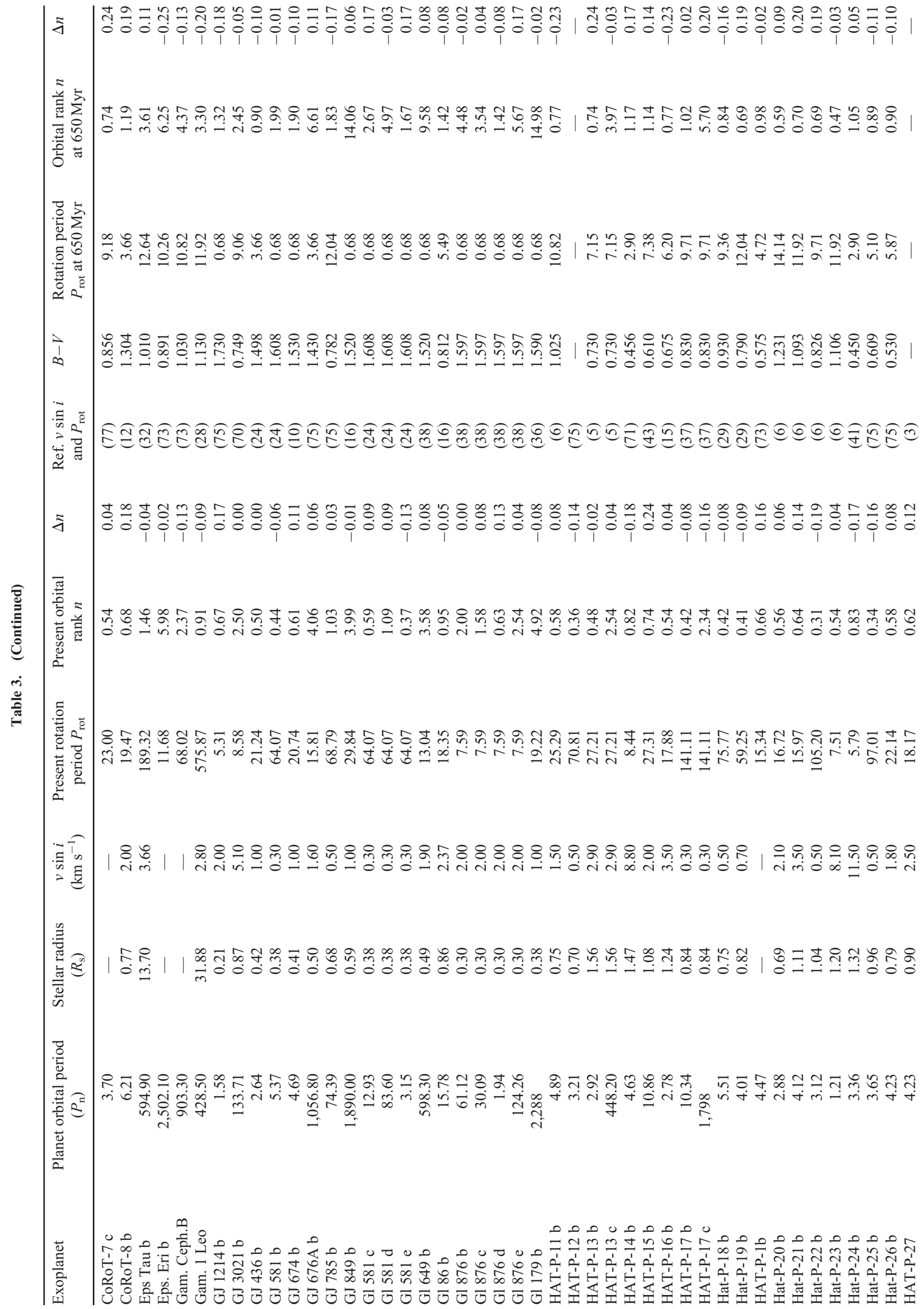




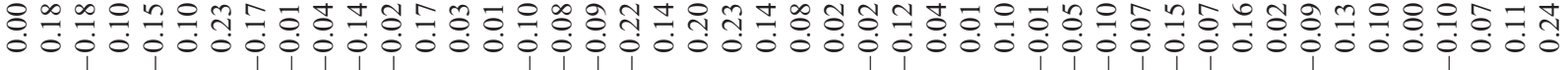

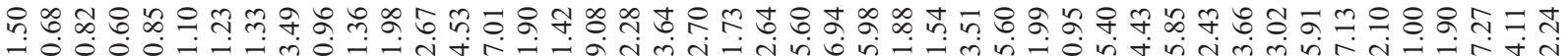

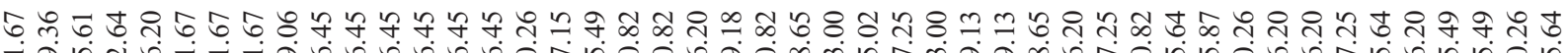
- an

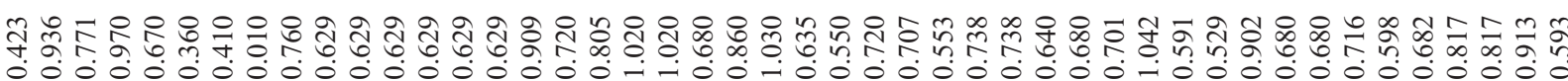

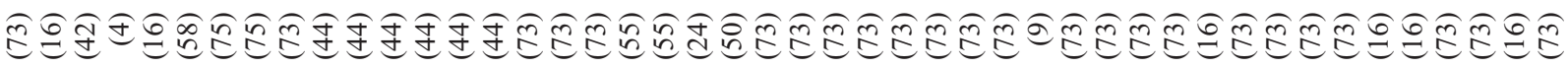

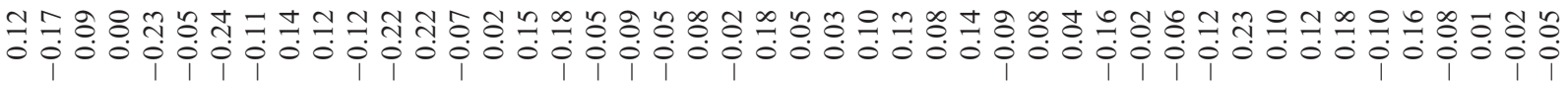

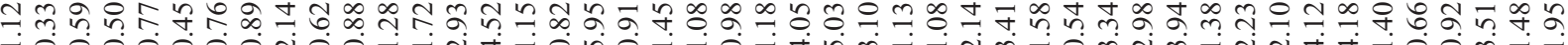

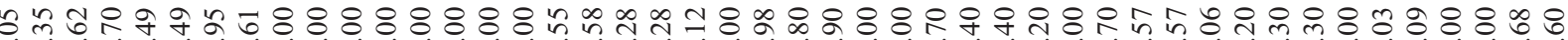

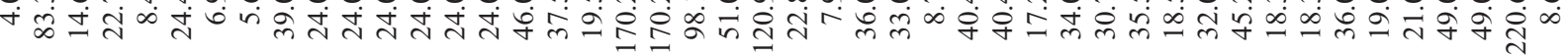

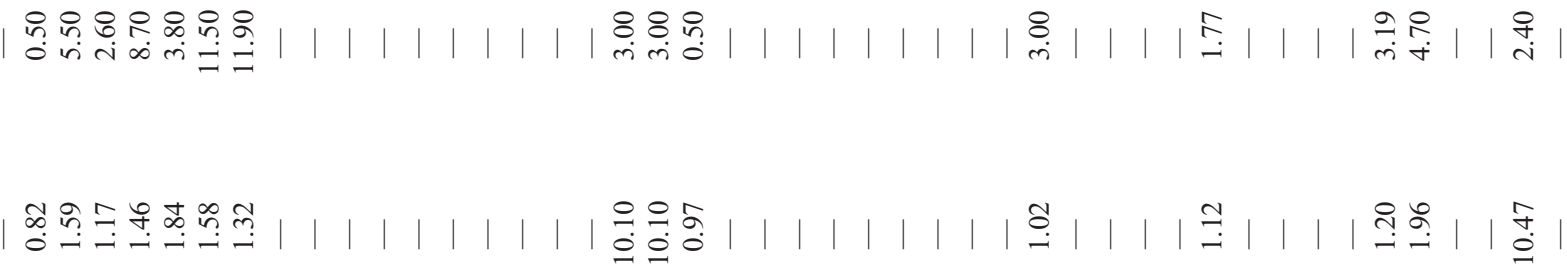

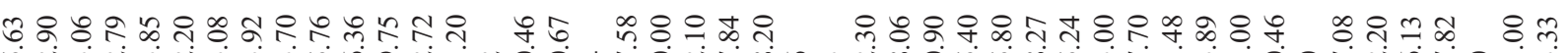

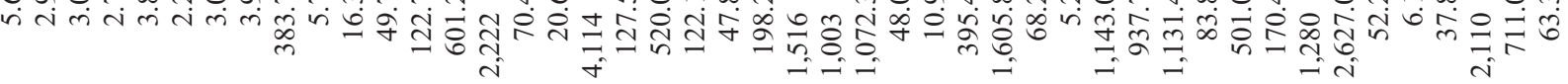




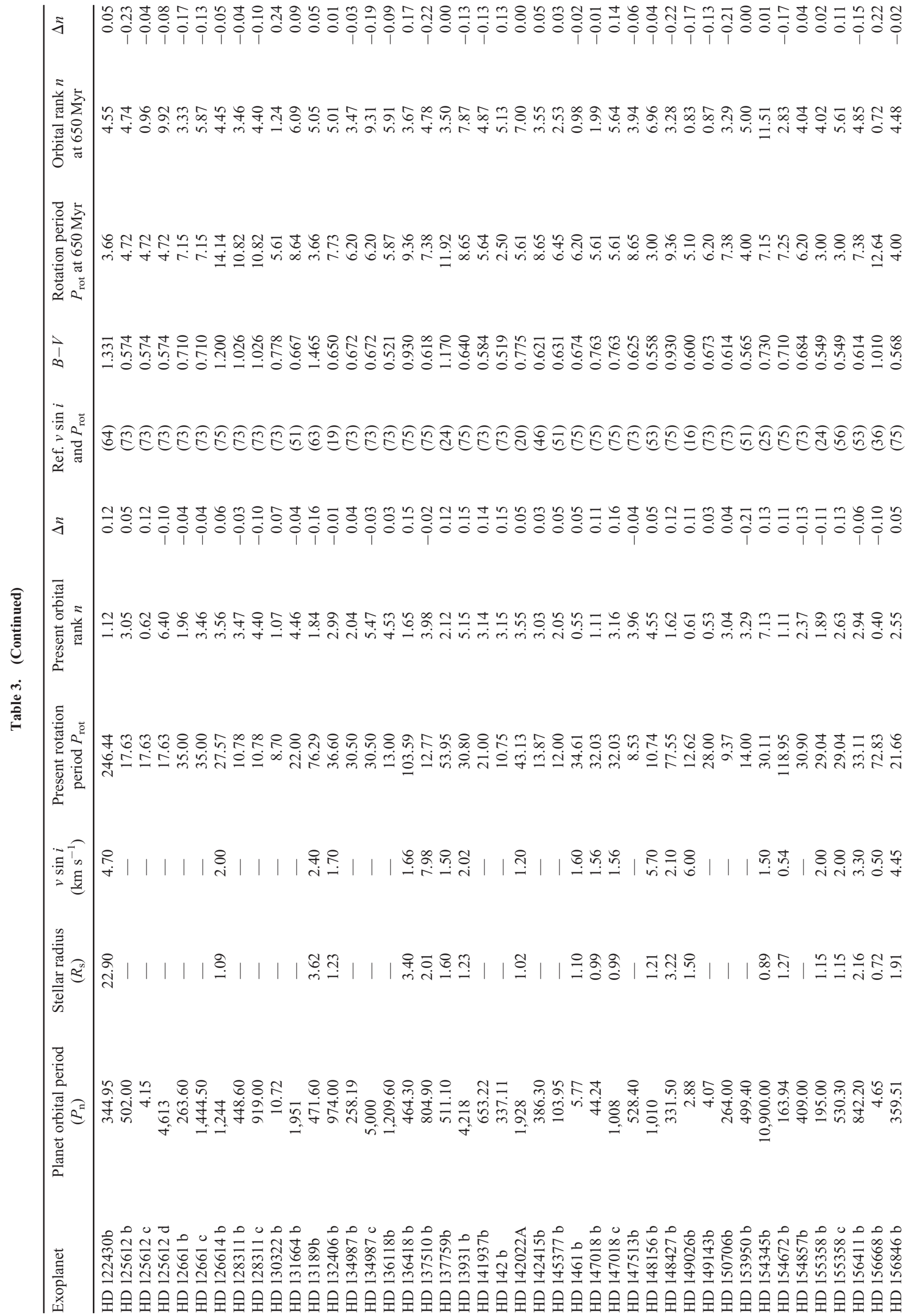




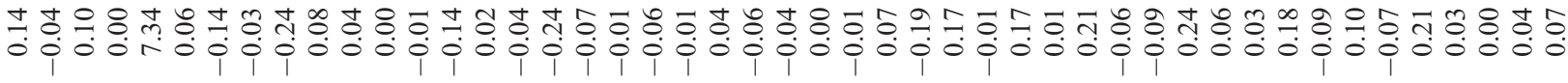

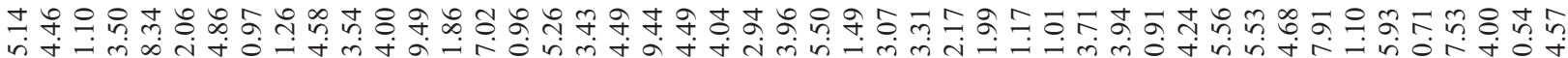

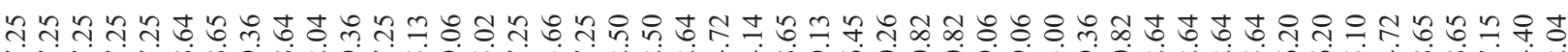

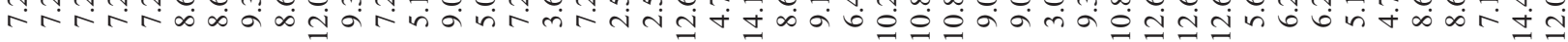

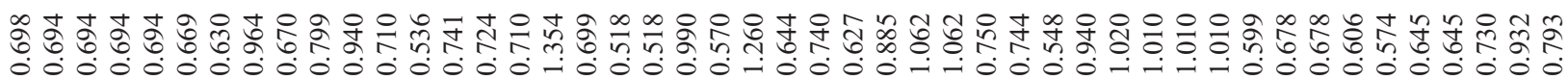

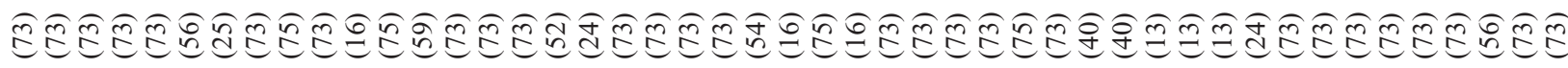
屍

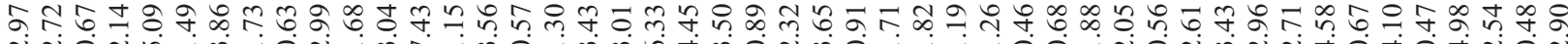

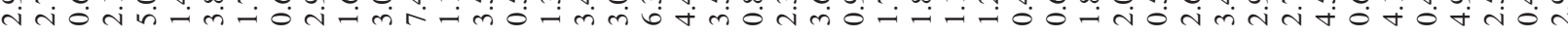

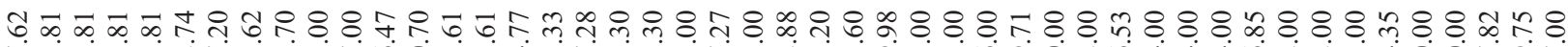

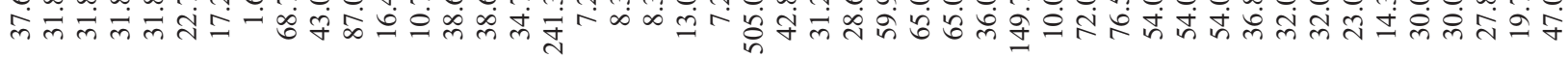

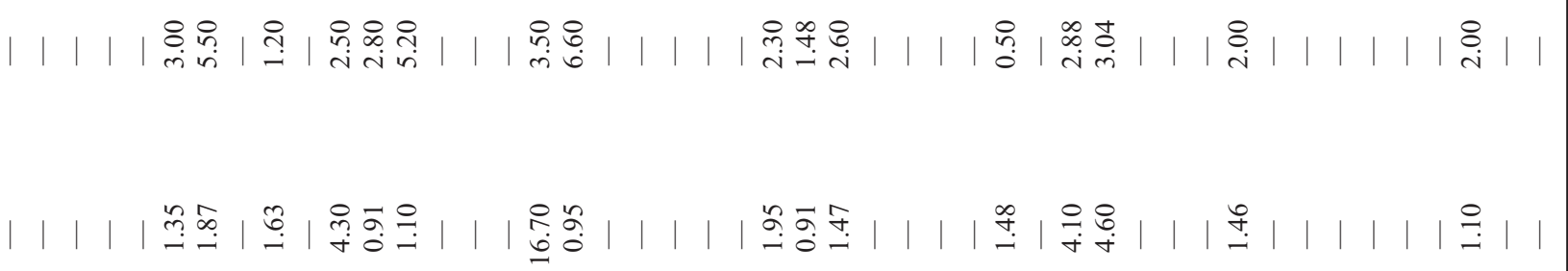

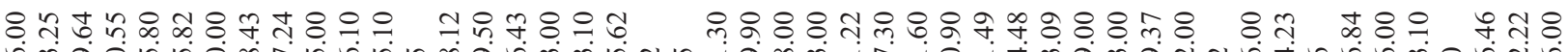

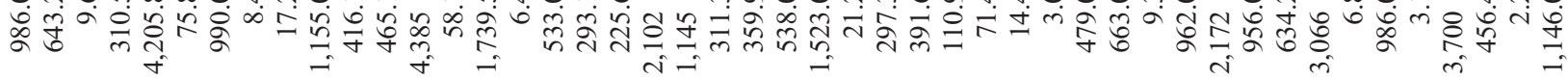

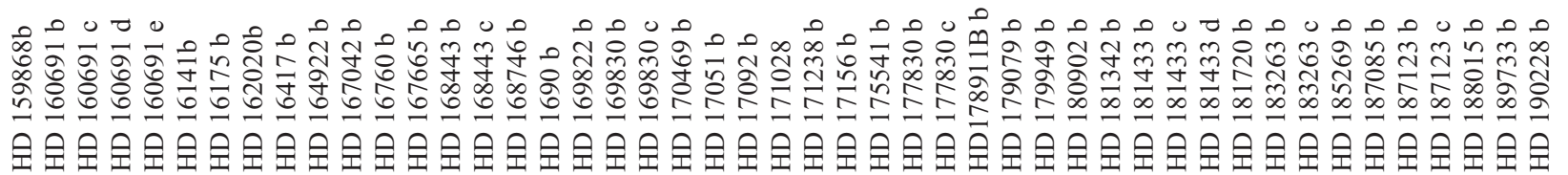




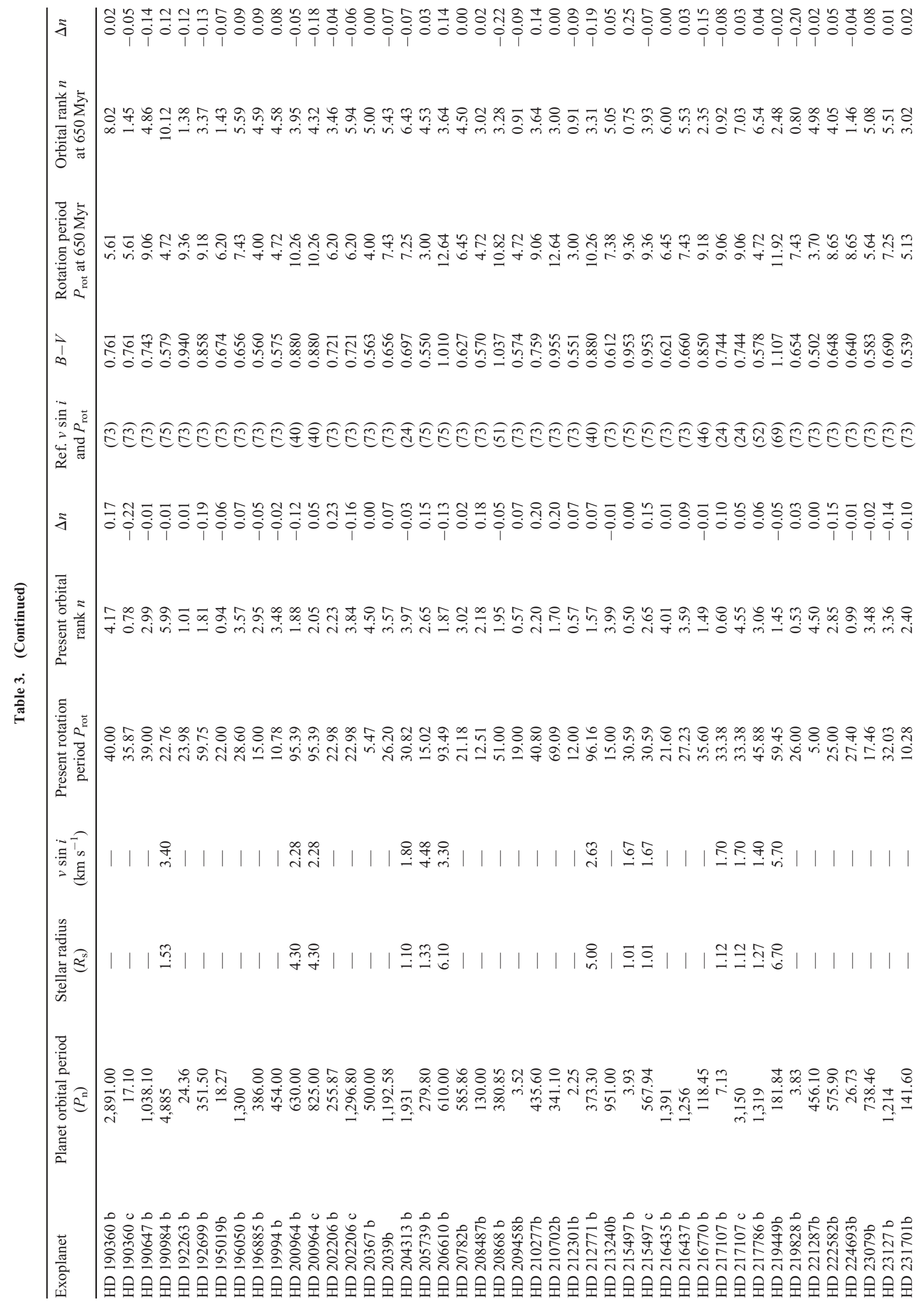




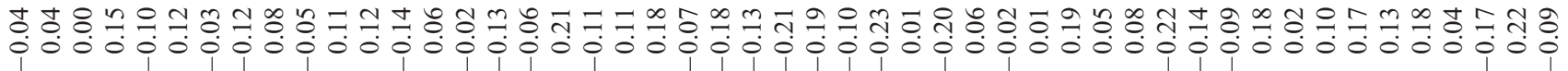

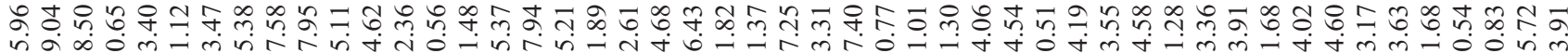
m

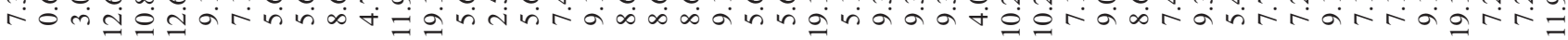

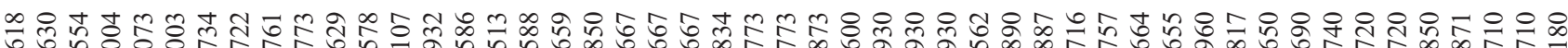
-

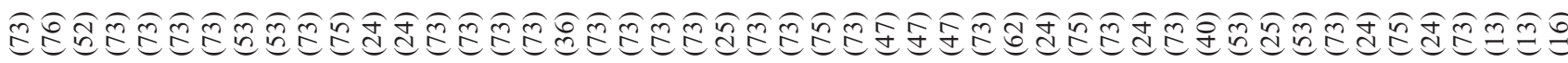

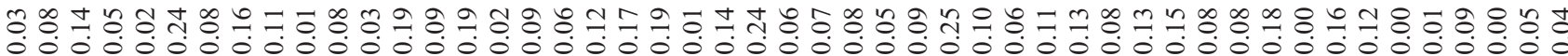

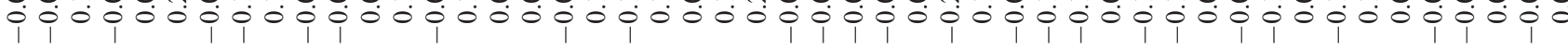

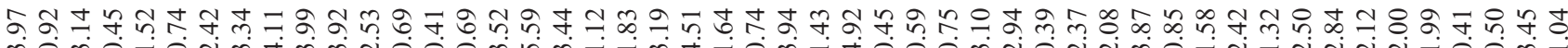

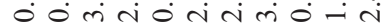

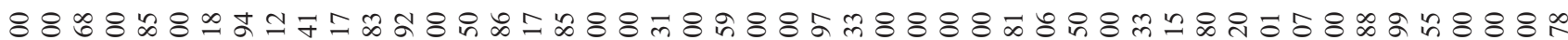

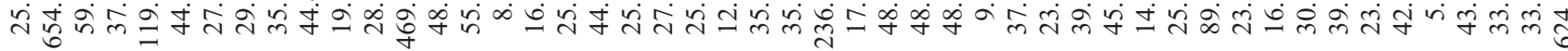

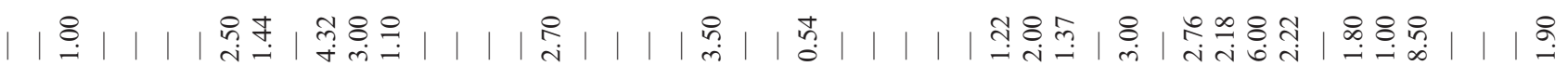

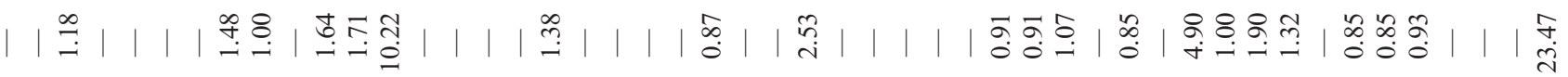

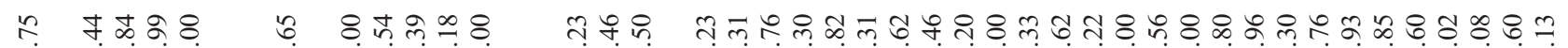

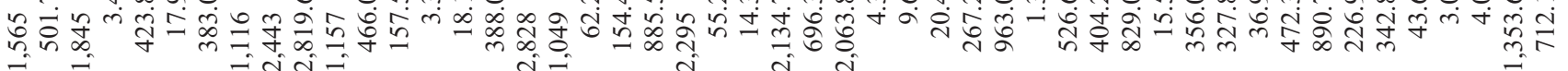

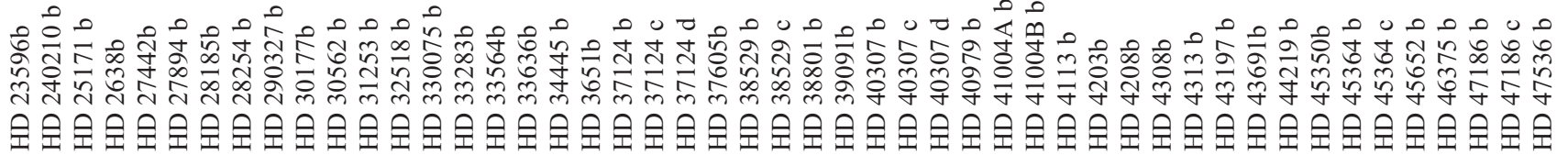




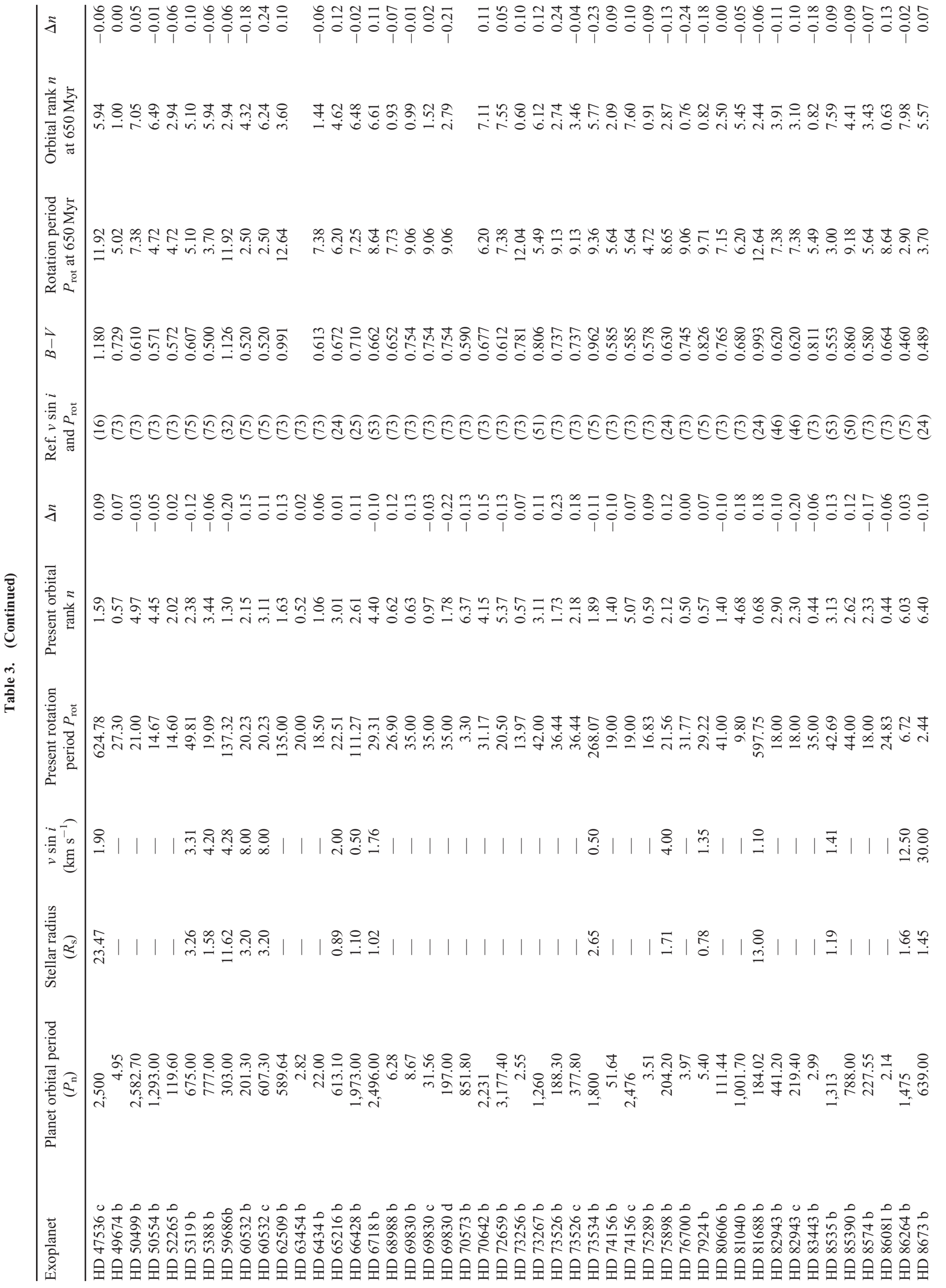




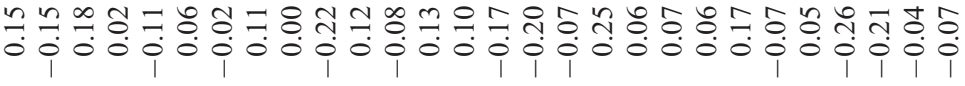

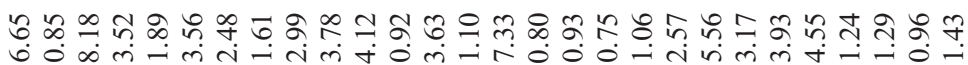

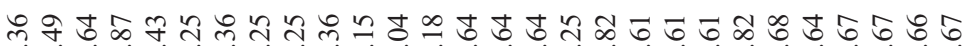

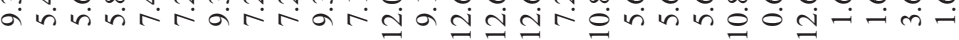

illo

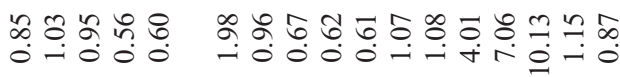

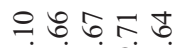

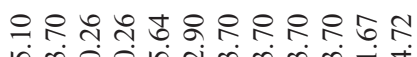

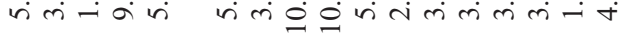

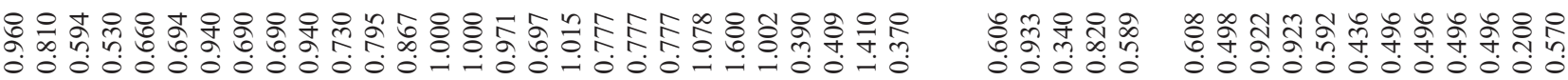

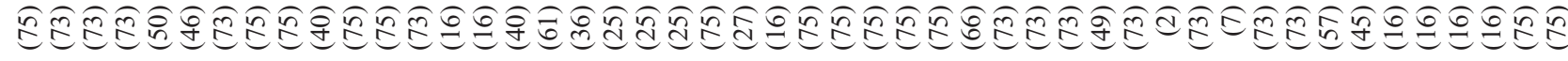

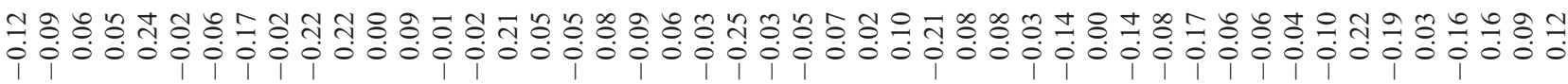

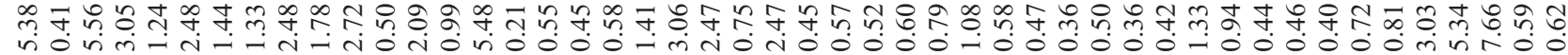

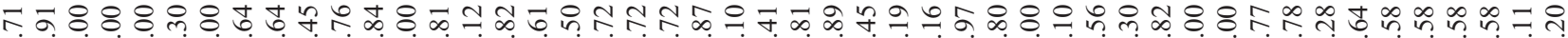

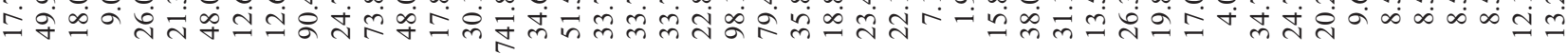

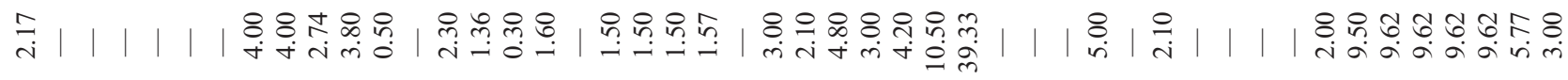

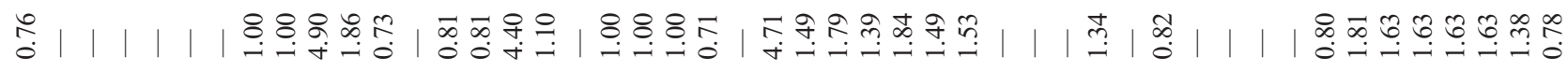

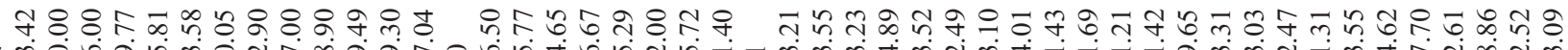

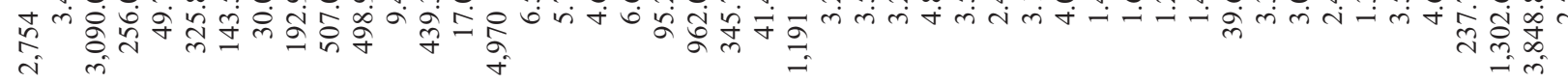

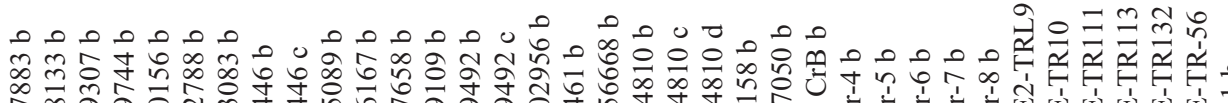

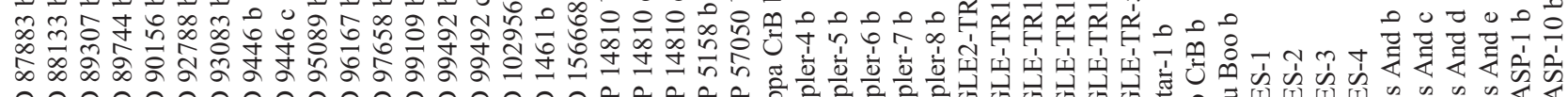

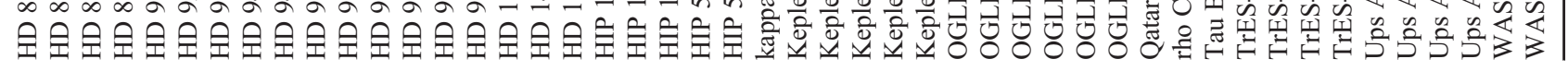




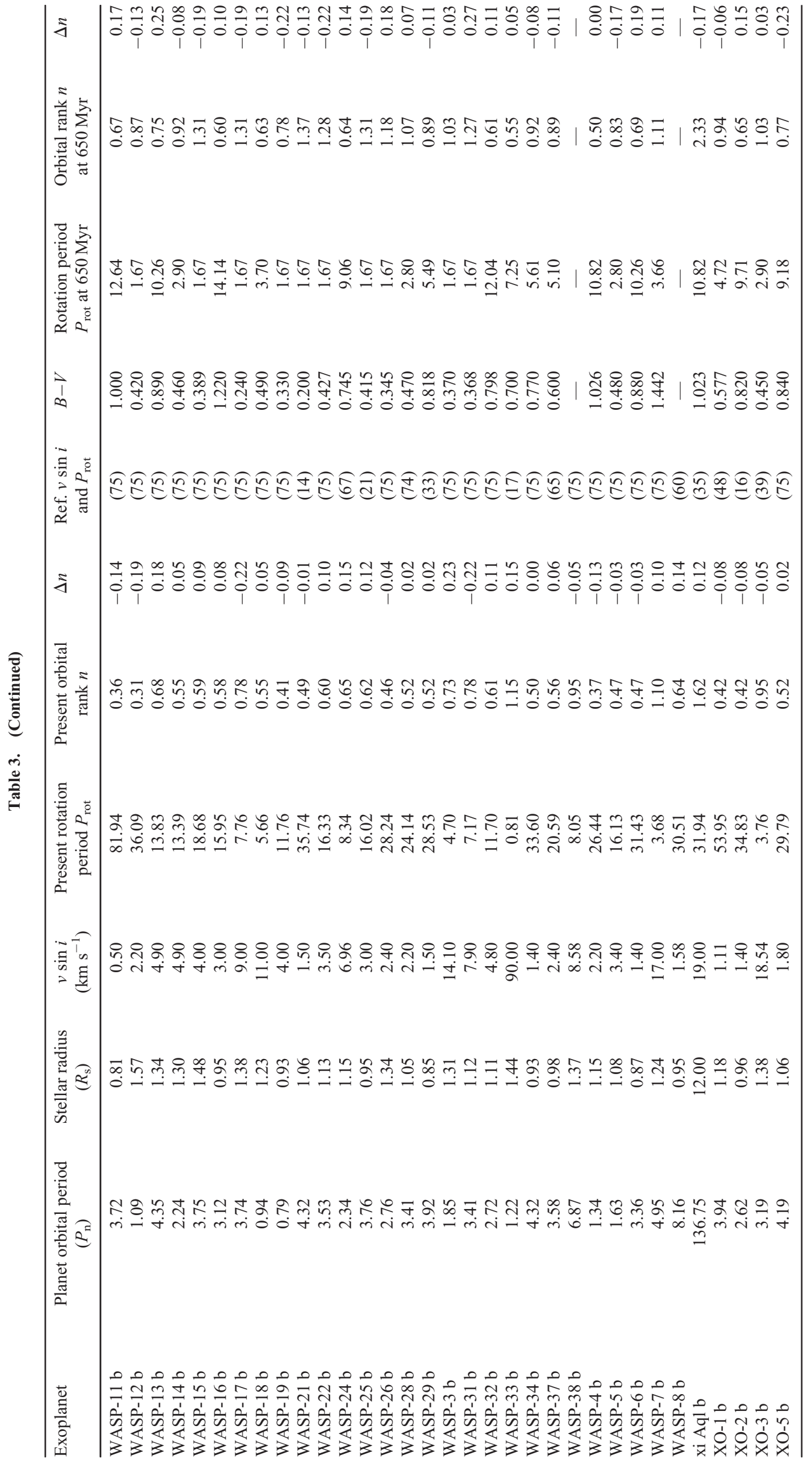

Oิ㇒

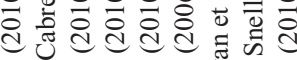

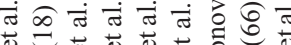

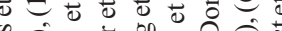

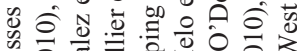

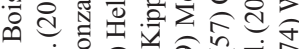

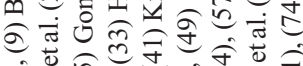

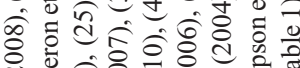
d बं

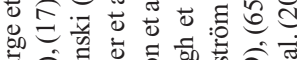

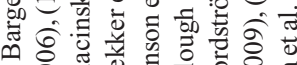
क⿺辶一兀

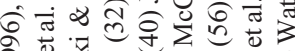

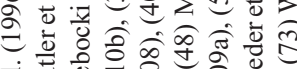

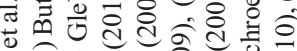

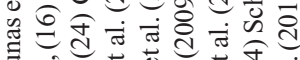
每 ๓̃ E d

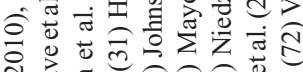

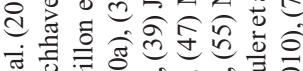

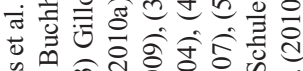

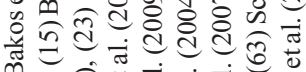
लै 6)

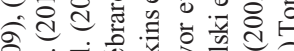

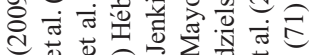

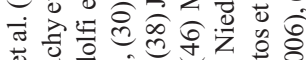

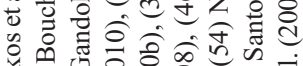

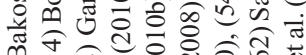
党过过

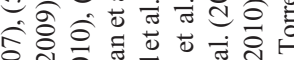

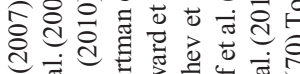
ส

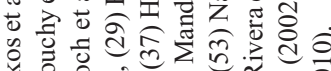

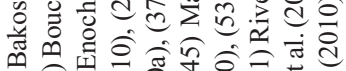

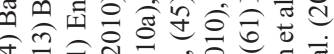
† 3 过

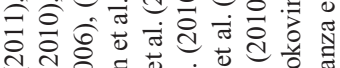
d d d

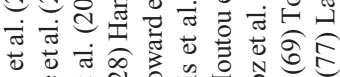

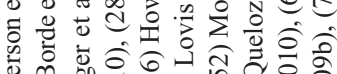

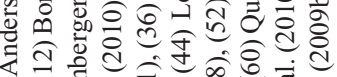
西 ○े

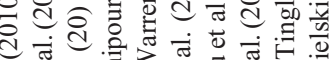

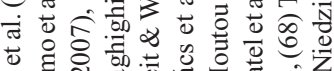

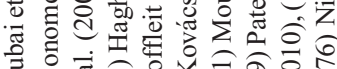

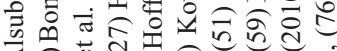
a

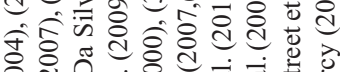

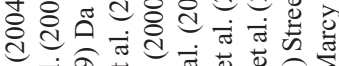
बं 20

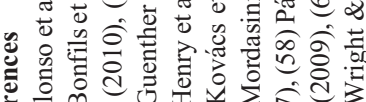

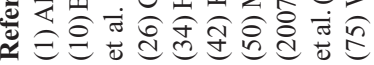


calculate the orbital ranks $n$ near the planet-formation epoch when the proposed quantization would have been established.

In Table 3, the orbital rank for each exoplanet is calculated using both the present stellar rotation period and the fiducial star rotation period at formation age (650 Myr) and the following is presented:

(1) The exoplanetary orbital periods, obtained from the updated online database of the Exoplanets Encyclopedia website ( Schneider $^{1}$ );

(2) The present orbital ranks $n$ and deviations from halfinteger $\Delta n$, calculated from the current stellar rotation periods at present, either directly measured $P_{\text {rot }}$ or derived from $v \sin i$ and the stellar radii (see $v \sin i$ and $P_{\text {rot }}$ references);

(3) The fiducial orbital ranks $n$ at formation age (650 Myr) and deviations from half-integer $\Delta n$, calculated from directly measured rotation periods at the fiducial age of $650 \mathrm{Myr}$ from Hyades stars having $B-V$ values that match those of the exoplanetary stars (Table 2 above).

\section{Statistical Analysis of Quantization Results}

\subsection{Statistical Rest for $\Delta n$ Deviations from Half-integer Values}

From Section 4.1, the distribution of the orbital ranks $n$ for the 443 exoplanets (in bins of 0.1 increment) that were calculated for the present stellar rotation periods is presented in Fig. 1, along with the distribution of deviations $\Delta n$ from half-integers in Fig. 2. It can be observed that the orbital ranks $n$ indeed tend to cluster around integer or half-integer values, similar to the Solar System results. Around 288 exoplanets (65 percent) have absolute deviations of $|\Delta n|<0$.1. If there is no correlation between the stellar rotation periods and the planetary orbital periods, the deviations from half-integer $\Delta n$ would follow a uniform distribution as opposed to the distribution observed in Fig. 2. To test that the results were not obtained by chance, we therefore need to reject the null hypothesis corresponding to a uniform distribution of deviations $\Delta n$ in the interval $[-0.25,0.25]$. A KolmogorovSmirnov one-sample test between the observed cumulative $\Delta n$ distribution and that of a uniform distribution yields a maximum difference statistic $D=0.54$ and a probability $p<0.046$ to have been obtained by chance. Using a $\chi^{2}$ test with 20 bins, we obtain $\chi^{2}=32.89$ and the probability for $\Delta n$ to be drawn from a uniform distribution, i.e. the probability for the results to be obtained by chance is $p<0.024$

\subsection{Monte Carlo Simulation for Uncertainty in Present $\mathrm{v}$ sin $\mathrm{i}$ and Rotation Periods}

To address the uncertainty in rotation periods derived from stellar radii and $v \sin i$, we have used a Monte Carlo

\footnotetext{
${ }^{1}$ The Extrasolar Planets Encyclopedia: Interactive Extrasolar Planets catalog. http://exoplanet.eu/catalog.php
}

treatment and calculated the orbital ranks from 25 randomly generated rotation periods ranging within \pm 20 per cent of the estimated rotation period for each of the 443 exoplanetary stars and resulting in a total of 11,075 simulated orbital ranks. The Monte Carlo distribution of orbital ranks is presented in Fig. 3 and their deviation from half-integer $\Delta n$ is presented in Fig. 4 . We note a clustering of orbital ranks around half-integers for $n=0.5,1.0$, and 1.5 , representing around 50 percent of all exoplanets, while the distribution of orbital ranks beyond $n>2.0$ starts to resemble a uniform distribution. This is expected since the uncertainty in rotation periods, discussed in Section 4.1, was expected to result in a 10 percent uncertainty in orbital ranks $n$, which in turn exceeds the critical absolute deviation $|\Delta n|=0.25$ for orbital ranks higher than $n>2.0$. Nonetheless, a Kolmogorov-Smirnov one-sample test between the observed cumulative $\Delta n$ distribution and that of a uniform distribution yields a maximum difference statistic $D=0.54$ and a probability $p<0.046$ to have been obtained by chance.

\subsection{Statistical Test for $\Delta n$ Distribution Using Estimated Rotation Periods at Formation Age}

Using the alternative approach of Section 4.2, the distribution of orbital ranks calculated from the stellar rotation periods at the formation epoch, which were derived from the $B-V$ colour of Hyades stars at the fiducial age of $650 \mathrm{Myr}$, is presented in Fig. 5 and the distribution of deviations from half-integer values is presented in Fig. 6. Clustering around half-integers is again apparent. If the results were obtained by chance, the deviations from halfinteger $\Delta n$ would follow a uniform distribution. However, out of the 443 exoplanets in the sample, 252 planets (57\%) have $|\Delta n|<0.1$. The mean absolute deviation from integer or half-integer values is $|\Delta n|=0.09$ with a standard deviation of 0.05 . The average deviations taken as a percentage of the orbital ranks, $\Delta n / n$, is 5 per cent. A Kolmogorov-Smirnov one-sample test between the observed cumulative $\Delta n$ distribution and that of a uniform distribution yields a maximum difference statistic $D=0.54$. For the 443 data points, this result has a probability $p<0.046$ to be obtained by chance. Using a $\chi^{2}$ test with 11 degrees of freedom (bins), we obtain $\chi^{2}=57.4$ and the probability for $\Delta n$ to be drawn from a uniform distribution, i.e. the probability for the results to be obtained by chance, is $p<2.8 \times 10^{-8}$.

\subsection{Monte Carlo Simulation for Uncertainty in Estimated Rotation Periods at Formation Age}

According to Radick et al. (1995), the measured rotation periods for Hyades stars vary between 2 to 8 per cent and have an accuracy of \pm 0.1 day. We have taken the worst case and considered 8 per cent uncertainty in all measured rotation periods, which translates to around \pm 1 day for the majority of the stellar rotation periods. To address this inherent uncertainty, we apply a Monte Carlo treatment 


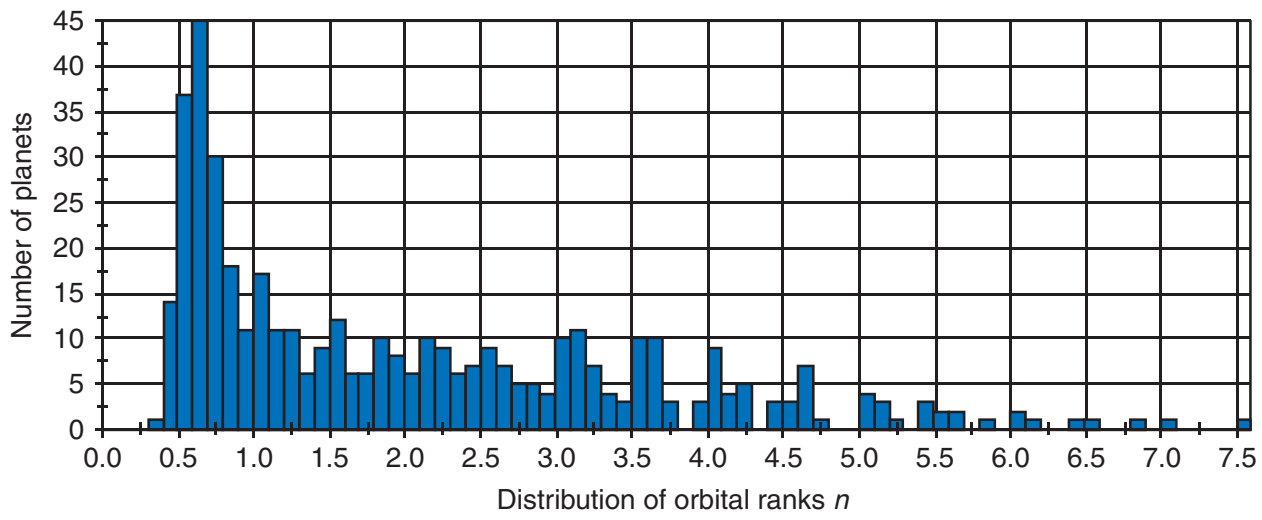

Figure 1 Distribution of present orbital ranks $n$ for the 443 exoplanets indicating an obvious clustering around discrete half-integer values, with peaks at $n=0.5,1.0,1.5,2.0,3.0,3.5,4.0,4.5,5.0,6.0,6.5$.

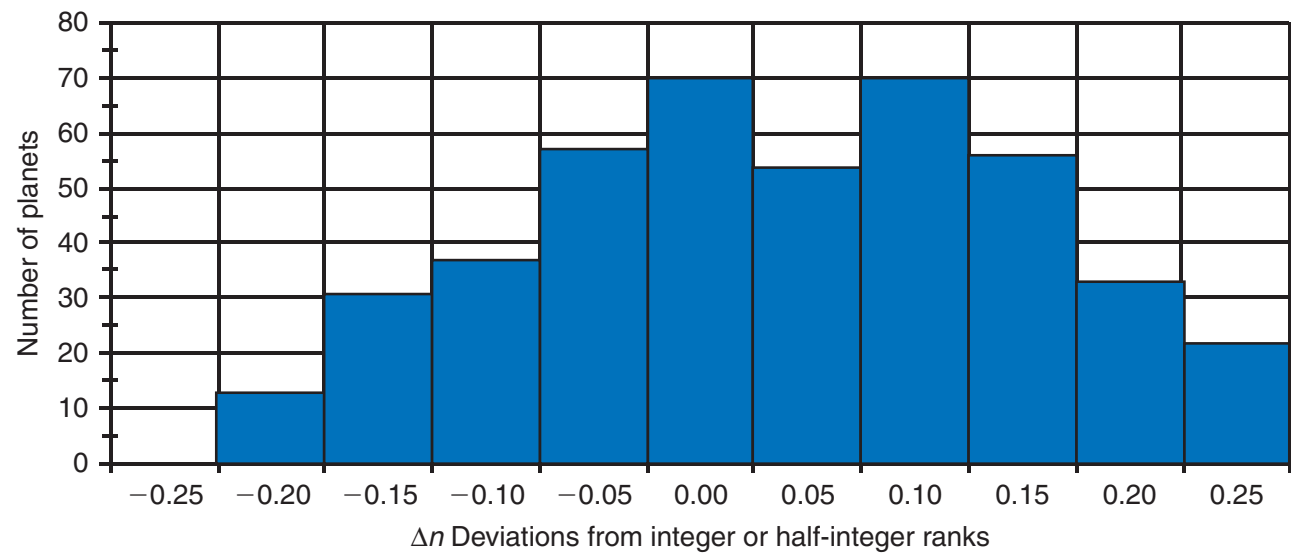

Figure 2 Distribution of $\Delta n$ deviations from the nearest half-integer for the 443 exoplanets, indicating that $65 \%$ of deviations are within \pm 0.1 of the nearest half-integer. The probability to obtain these results by chance is $p<0.024$.

and calculate the orbital ranks $n$, using 50 randomly generated rotation periods (within \pm 8 per cent of the measured rotation periods) for each of the 443 exoplanetary stars, resulting in a total of 11,075 orbital ranks. The Monte Carlo distribution of orbital ranks and their deviation from half-integer $\Delta n$ is presented in Fig. 4 . Again, a clustering around half-integers is obvious, all the way up to orbital ranks $n<6$, representing around $90 \%$ of all exoplanets. A Kolmogorov-Smirnov one-sample test yields a maximum difference statistic $D=0.52$ between the observed cumulative distribution and that of a uniform distribution. For the 11,075 data points, this result has a probability $p<0.0035$ to be obtained by chance. Using a $\chi^{2}$ test with 20 degrees of freedom (bins), we obtain $\chi^{2}=1,835$ and the probability for the results to be obtained by chance is nil.

\subsection{Test for Mathematical Artefacts}

In order to investigate the origin of the apparent quantization in Figs. 1 to 7, we shuffled the stellar and planetary angular velocities, to give randomised matches between the properties of the stars and the properties of the planets, to see whether the quantization persists. As a result, the
$\Delta n$ deviations gave a uniform distribution in the interval $[-0.25,0.25]$, and the quantized distribution disappeared, which again supports the hypothesis that quantization did not occur by chance.

It is important to note that while the obtained quantization results are valid for stellar rotation periods both at present and at the formation age, it is observed that the tendency to cluster around half-integers seems to improve and become more pronounced as stellar rotation $P_{\text {rot }}$ increases and rotation slows down. The mean absolute $|\Delta n|$ deviation from half-integer values is 0.1 for rotation periods derived at the present age and is also the same value for those derived at the formation age. However, the standard deviation for $|\Delta n|$ derived from present rotation periods is 0.068 and is improved compared to the standard deviation of 0.075 for $|\Delta n|$ derived from estimated rotation periods at formation age. Additionally, the $|\Delta n|$ distribution's peak at formation age is more flat (with a positive kurtosis $k=0.97$ ) and becomes much sharper (with a negative kurtosis $k=-0.71$ ) as rotation slows down to the present values, i.e. the quantization features improve.

The distribution of the ratio $r$ of the planetary orbital period to the stellar rotation period is not uniform, but 


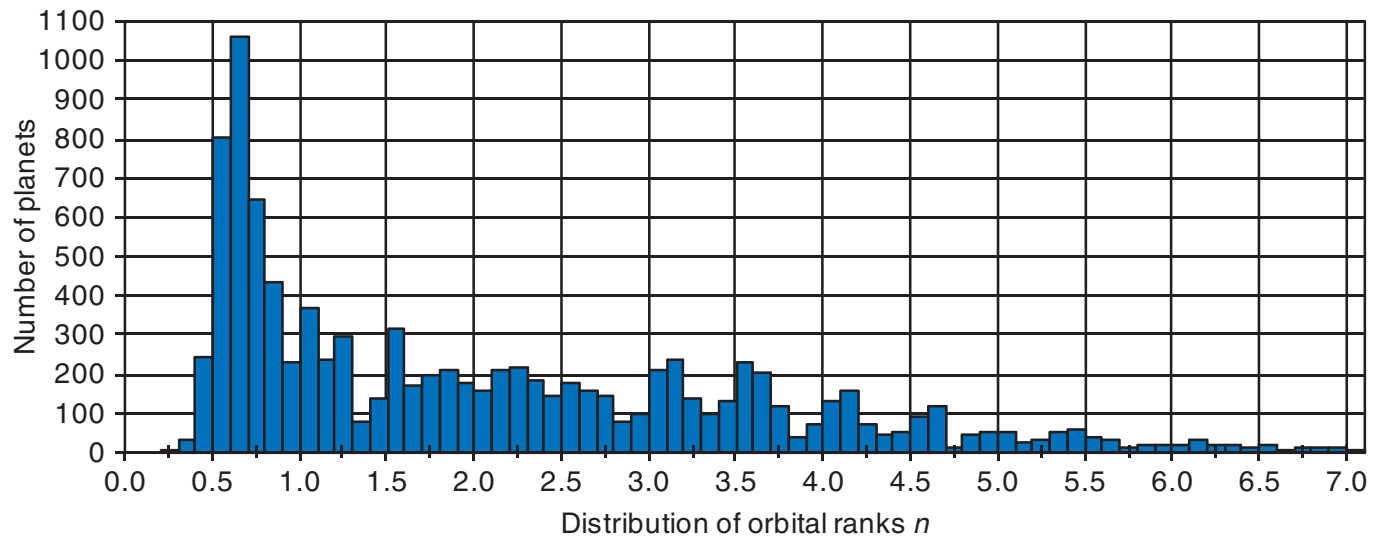

Figure 3 Distribution of orbital ranks $n$ calculated from 25 randomly generated rotation periods (within 20\% uncertainty) for each of the 443 exoplanets, indicating a clustering around discrete half-integer values with obvious peaks at $n=0.5,1,1.5,3.0,3.5,4.0,4.5,5.0$.

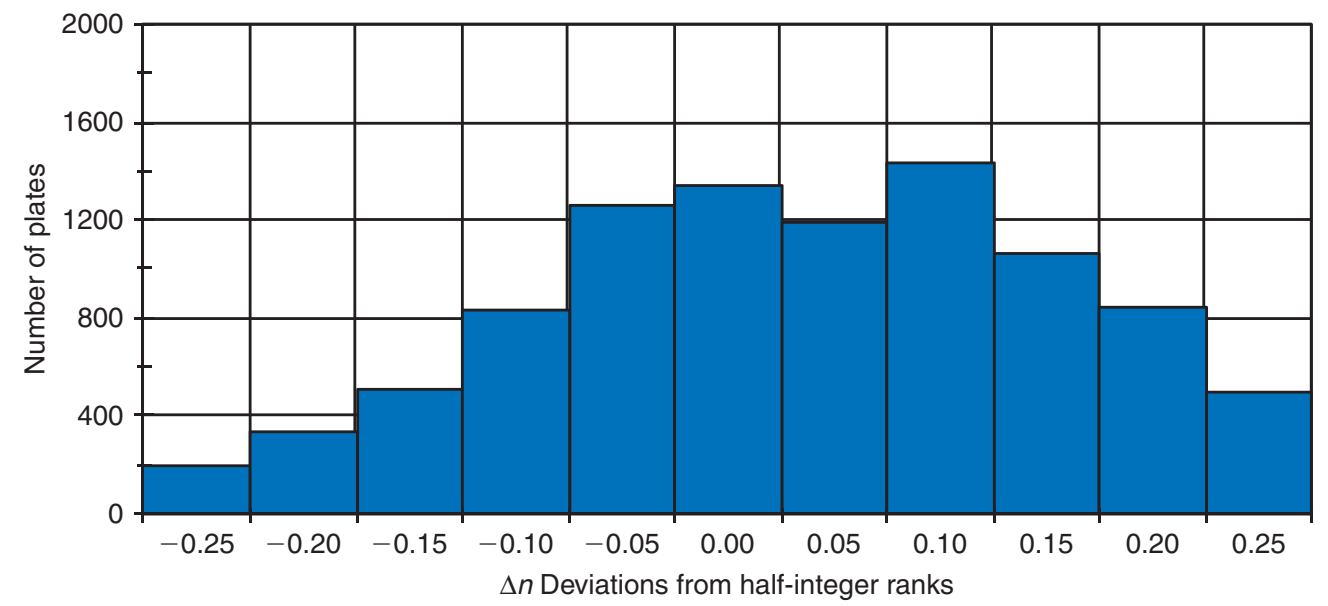

Figure 4 Distribution of deviations from half-integer values $\Delta n$ calculated from the 25 randomly generated rotation periods (within $20 \%$ uncertainty) for each of the 443 exoplanetary parent stars. The probability to obtain these results by chance is $p<0.046$.

decreases with increasing $r$ due to the bias in detecting exoplanets with short orbital periods. In order to test whether the above quantization result is fundamental in nature or whether it is simply the result of this selection effect, the following test was proposed. A continuous 'toy' distribution of $r$ is used which has the gross properties of the actual distribution, that is, it reflects the observational biases rather than real biases. A histogram for the distribution of orbital ranks $n$ is then generated using the same method as in Section 4, i.e. using the cubic root relationship of Equation (9). We then searched to see whether any quantization features persisted, which would support the hypothesis that they are not fundamental in nature, but effectively a mathematical artefact. However, as shown in Fig. 8, the quantization features almost disappeared and no major clustering around half-integer was observed except for a peak at 0.6 , which was expected due to the large number of short-period planets observed. The distribution around the peak at $n=0.6$ is gradual, uniform, and not as sharp as the distribution around $n=0.5$ in Fig. 1 and Fig. 3. Moreover, the original peaks in Fig. 1 and Fig. 5 at $n=1.0$,
1.5, 2.0, 3.0, and 3.5 have almost disappeared in Fig. 8. This implies that the hypothesised quantization is not the result of mathematical artefacts and must have some fundamental physical basis.

\subsection{Statistical Analysis Summary}

In summary, if the planetary orbital periods are not quantized with respect to the stellar rotation period, the orbital ranks $n$ and their deviation $\Delta n$ from half-integer values would have exhibited a uniform distribution. However, the peaks in orbital ranks $n$ at half-integer and the observed distribution in $\Delta n$, indicates that this is not the case and that the probability to obtain such a distribution by chance is $p<0.024$. This was done for orbital ranks derived both from present rotation periods and from those estimated at the formation age ( $650 \mathrm{Myr})$.

The uncertainties in $v \sin i$ and rotation periods were dealt with using a Monte Carlo treatment by generating random rotation periods within a range of 20 per cent of the estimated rotation periods. The derived distribution of orbital ranks continued to show some quantization peaks, 


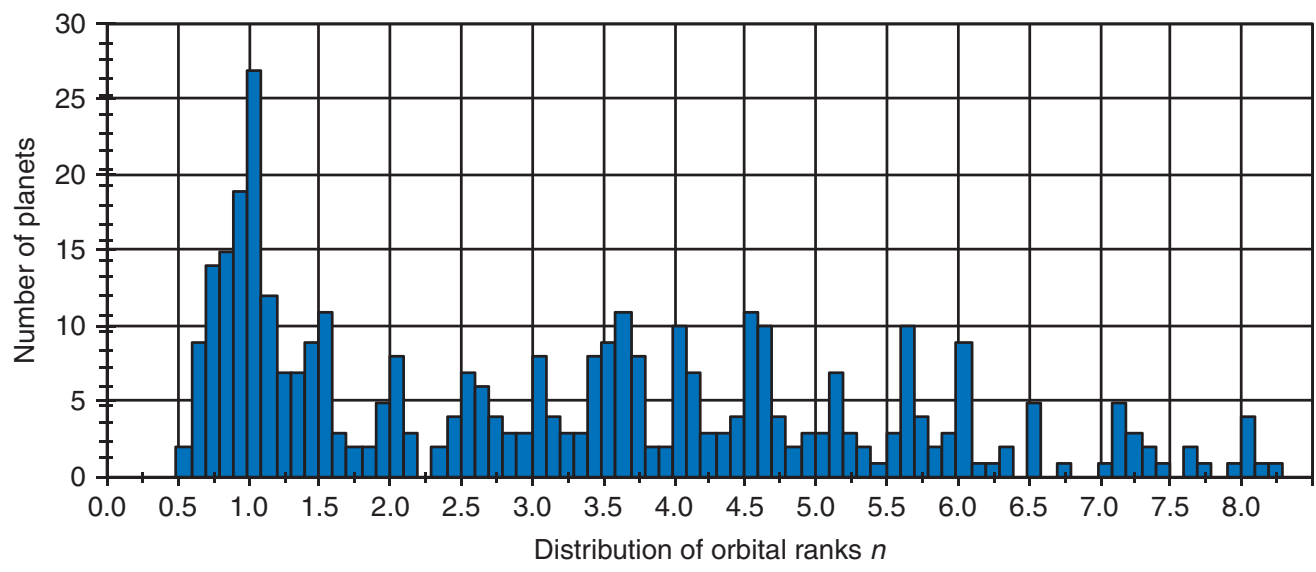

Figure 5 Distribution of orbital ranks $n$ for the 443 exoplanets, calculated from rotation periods of matching Hyades stars at the fiducial age of $650 \mathrm{Myr}$, again indicating an obvious clustering around discrete integer or halfinteger values.

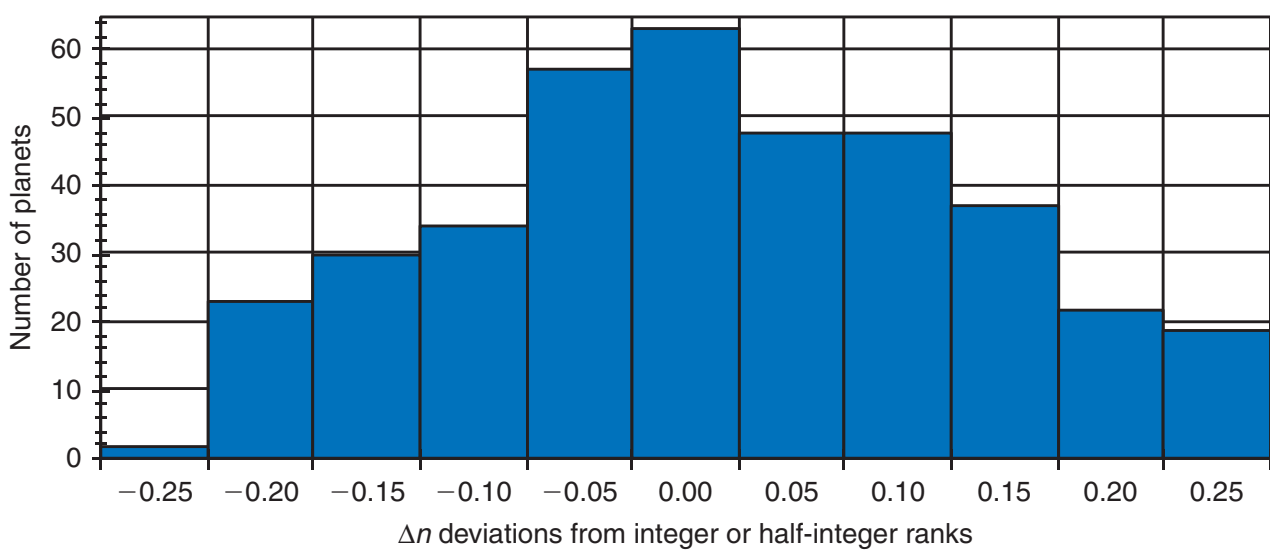

Figure 6 Distribution of $\Delta n$ deviations from the nearest half-integer for the 443 exoplanets, calculated using the matching Hyades stars at formation age of $650 \mathrm{Myr}$. The probability to obtain these results by chance is $p<2.8 \times 10^{-8}$.

which were, however, less pronounced. The test for mathematical artefacts proves that a uniform distribution of $r$, i.e. the ratio of planetary orbital periods to the star's rotation period, does not produce the hypothesised quantization results.

In the above statistical analysis, we did not include the Solar System results, for which the planetary orbital periods and the Sun's rotation period are accurately determined. Regardless of the uncertainties in stellar rotation periods, the orbital ranks $n$ in the Solar System are clearly quantized over half-integer values and this provides additional support to the quantization hypothesis.

\section{Discussion}

\subsection{Quantized Orbits Featuring in Hybrid Models of Planet Formation}

The statistically significant results confirm the hypothesis that the specific orbital angular momenta of planets in the Solar System and extra-solar systems tend to be discrete and quantized, clustering around half-integer multiples of the specific angular momentum at the central star's corotation radius. This half-integer orbital quantization is therefore directly dependent on the parent star's rotation rate and is not related to any universal constant, but is a system-specific physical property. This provides a more physical description of the Titus-Bode empirical law found in the Solar System and possibly some extra-solar multi-planetary systems, which can now be interpreted using a quasi-quantum physical model with stellar rotation as the main quantization parameter.

One possible theoretical justification for having halfintegers as the fundamental unit of quantization and not some other fraction, such $1 / 3,1 / 5$, or even integer multiples, might have to do with the similarity of the planetary quantization with the Bohr-Sommerfeld atomic quantum model. The planetary quantization and its dependency on stellar rotation is consistent with a corresponding dependency at the atomic scale, where the discrete angular momenta of electron orbits are integer multiples of 

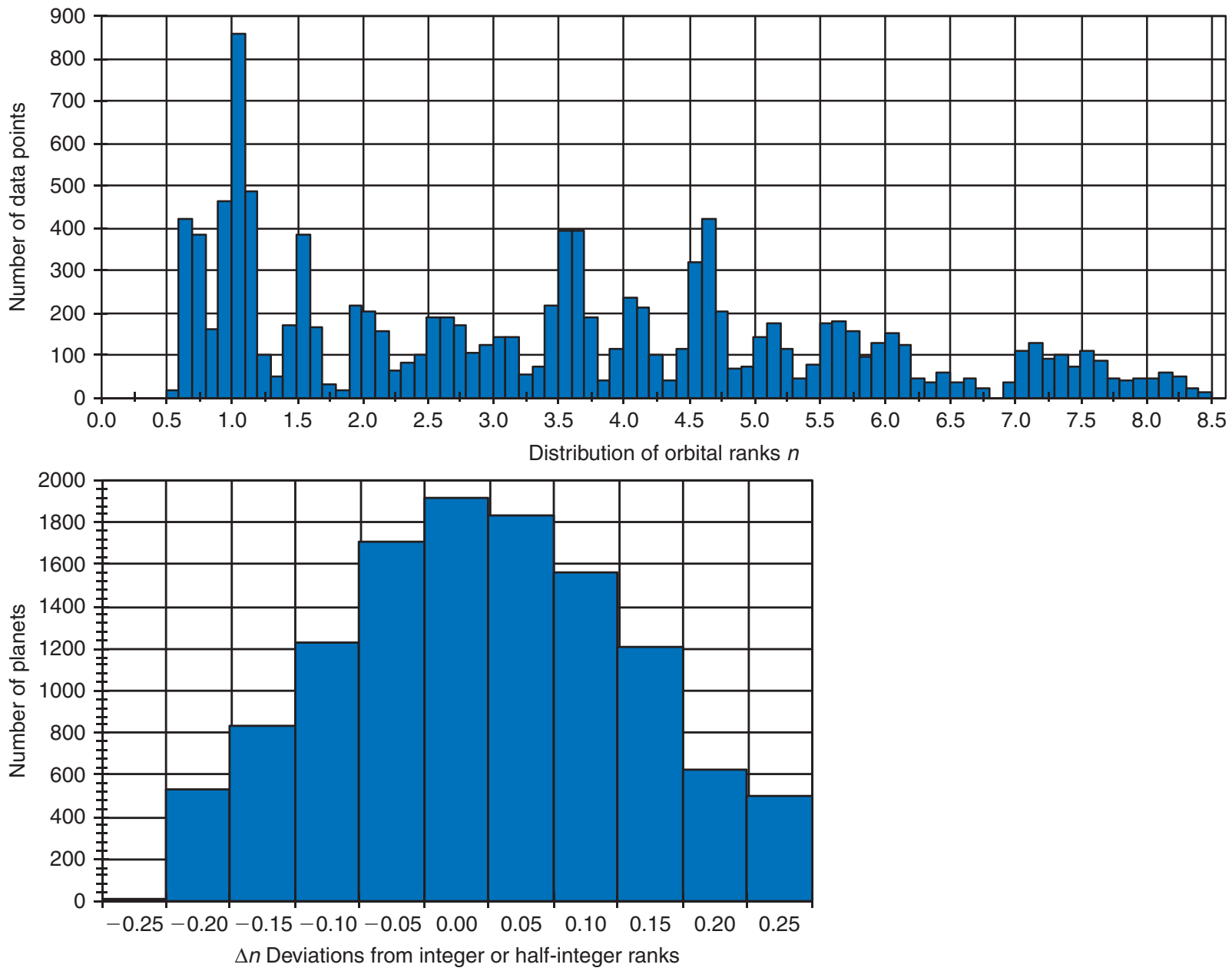

Figure 7 Distribution of orbital ranks $n$ calculated from 50 randomly generated rotation periods (within $8 \%$ uncertainty) for each of the 443 exoplanets, indicating an obvious clustering around discrete integer or half-integer values. The probability to obtain these results by chance is almost nil.

Planck's reduced constant $\hbar$ and the spin angular momentum is in discrete multiples of $1 / 2 \hbar$. Regardless of the theoretical justification, the study shows that this planetary quantization, with its dependency on stellar rotation, is better explained as a product of physical mechanisms involved in the planet-formation process.

The diverse properties of exoplanets, i.e. orbital semimajor axes, orbital eccentricities, masses, and inclinations, are the result of a combination of events that took place during the initial formation stage (which includes possible migration and disc interactions), and the longerterm dynamical evolution stage which followed after the protoplanetary disc dissipated. It is not yet clear which of the two stages govern the shaping of the system's dynamical properties and long-term stability. Both current theories of planet formation, core accretion and gravitation instability, have their limitations (D'Angelo et al. 2010). The core-accretion model suffers from timescales that are too long for observed disc lifetimes and the gravitational instability model has some difficulties explaining the low disc temperatures needed for its operation. A new trend of hybrid models has emerged where the virtues of both models complement each other. In such hybrid models (e.g. Durisen et al. 2005), concentric dense gas rings created by gravitational instabilities enhance the growth rate of solid cores by drawing solids towards their centres, thus accelerating core-accretion and runaway growth (Haghighipour \& Boss 2003). Durisen et al. (2005) indicated that the dense rings appear to be produced by resonances with discrete spiral modes, which we suggest can be correlated with a quantum-like structure. The hybrid gravitational instability model of planet formation appears to be the best suited for explaining the reported quantization of planetary orbits. One reason is that the gravitational instability model has been successfully used in the past to explain 'discrete' power law distributions such as the mathematical regularity in planetary spacing observed in the empirical Titius-Bode law (Griv \& Gedalin 2005). Another reason is that it requires minimal orbital migration, at least initially, because the self-gravitating disc gas flows inward, past the protoplanets, leaving them relatively undisturbed (e.g. Boss 2005). This implies that planets can form directly in situ, or even by accelerated core-accretion, within the 


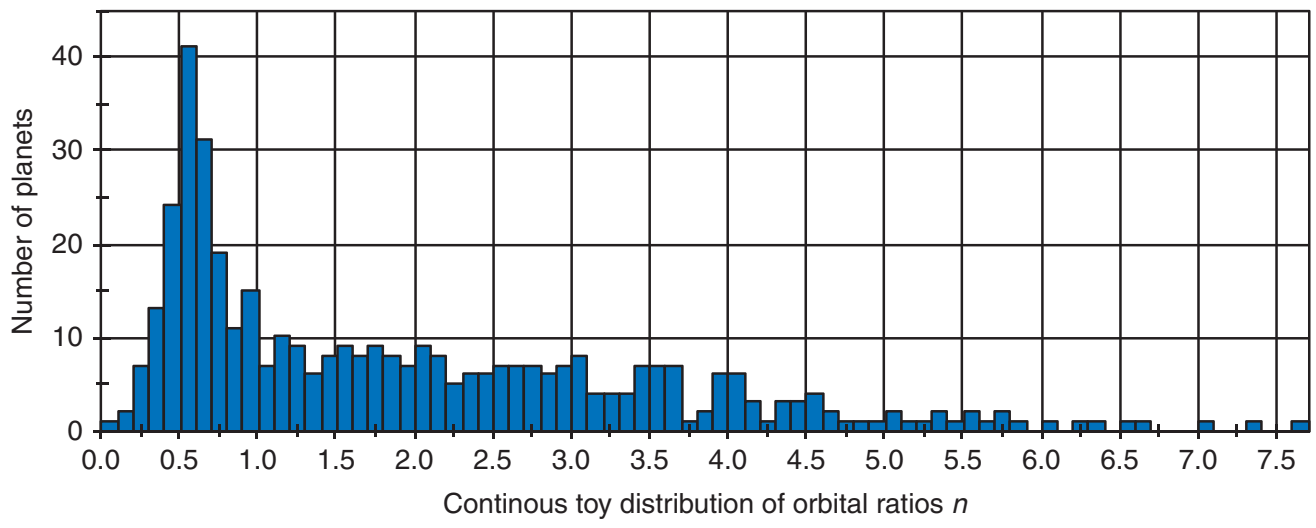

Figure 8 Distribution of orbital ranks $n$ calculated from a 'toy' distribution of $r$ ( $r$ being the ratio of planetary orbital period to the star's rotation period), showing insignificant quantization features, supporting the hypothesis that quantization is not the result of mathematical artefacts.

quantized spatial structures. However, even when protoplanets do migrate from their original birthplace, resonance can provide traps at discrete density jumps that enhance the accumulation of planetesimals (Masset et al. 2006) at these discrete 'quantized' locations.

\subsection{Disc Magnetospheric Truncation and the Lowest-ranking Orbits (at $\mathrm{n}=0.5$ )}

Various other natural mechanisms play a role in planetary structure and may explain the proposed quantization and its dependence on stellar rotation. One such possible mechanism is inner disc truncation by stellar magnetospheres (Lin \& Papaloizou 1996). The stellar magnetic field of a spinning star couples to the protoplanetary disc and expels ionized gas from its innermost part, carving an inner gap at the truncation radius in the range of 3-10 stellar radii, depending on the disc accretion rate and magnetic field strength, which in turn is a function of the star's rotation rate. A migrating planet that reaches this inner gap can remain parked there indefinitely, no longer being dragged in by the accreting disc nor forced to exchange angular momentum with it. Hence, the truncation radius at the disc's inner gap serves as a physical inferior limit for planetary orbits and acts as the planets' last line of defence against their fall into the star. Yi (1995) modelled magnetic braking and found that the final size of the truncation gap was in the range of 3-10 stellar radii and that radius is highly dependent on the stellar rotation period. Therefore, the orbital angular momenta of migrating planets that end up at their parent star's truncation gap should correlate well with the star's rotation period.

From the half-integer quantization results of Table 3 , the inferior limit of planetary orbits in any planetary system is at the discrete orbital rank $n=0.5$. In terms of the corotation radius $r_{0}$ and Equation (8), this inferior limit corresponds to a semi-major axis of $0.25 r_{0}$. In order to verify the relationship between the lowest ranking orbits and the stars' disc truncation radii, we calculated the semi-major axis $\left(0.25 r_{0}\right)$ of the lowest ranking orbit (at $n=0.5$ ) for each exoplanetary system under consideration. We found that the lowest ranking orbits range from 0.02 to $0.09 \mathrm{AU}$ with a peak and mean semi-major axis of $0.043 \mathrm{AU}(\approx 8$ mean stellar radii). The cumulative distribution, expressed in terms of the respective parent stars' radii, indicated that the lowest ranking orbits $(n=0.5)$ are clustered in the range 3-10 stellar radii, which is consistent with the predictions of protoplanetary disc models for magnetospheric inner gap sizes (e.g. Yi 1995). Hence, it is reasonable to suspect that the quantized lowest ranking orbits $(n=0.5)$ are physically described by the parent star's disc's inner gap size at the magnetospheric truncation radius. This is significant, since the correlation between the disc's inner gap size and stellar rotation (Yi 1995) provides further support for the dependency of the quantized lowest ranking orbits on stellar rotation.

\subsection{Tidal Dissipation and the Corotation Orbit $(\mathrm{n}=1.0)$}

Another mechanism that can explain the dependency of orbital ranks on stellar rotation is tidal dissipation. Lin \& Papaloizou (1996) suggested that as a migrating planet approaches the central star, it will raise tidal bulges in that star which will transfer angular momentum from the rapidly spinning star to the more slowly spinning planet. The tidal dissipation within the star can circularise the planet's orbit and synchronize its orbital period to the star's rotation. The resulting spin-orbit coupling can be effective at pushing the planet outwards, keeping it at or near the corotation radius. Indeed, observations indicate that planets within $0.1 \mathrm{AU}$ are nearly always on circular or nearly circular orbits, while beyond $0.3 \mathrm{AU}$ the distribution of eccentricities appears essentially uniform between 0 and 0.8 (Butler et al. 2006). This observed split in the eccentricity-period distribution is evidence of orbital circularization for short-period planets by internal tidal dissipation (Rasio et al. 1996). The tidal locking mechanism can therefore provide a natural physical justification for exoplanets with discrete orbital ranks $n=1$, since by definition these planets are located at the corotation orbit. 
Recently, Alves et al. (2010) confirmed that the angular momentum of exoplanet parent stars follows, at least qualitatively, Kraft's relation. The mechanism of angular momentum transfer must then certainly have a role in producing this apparent quantization in the exoplanets angular momentum distribution.

\subsection{Hot Jupiters and Multi-planetary Sample Analysis}

The sample of 443 exoplanets can be split into 2 samples: 75 'hot Jupiters' and 368 'non-hot Jupiters'. We analysed each sample separately to see whether the quantization features would be different for systems with hot Jupiters. In both samples, the distribution of deviations from halfintegers remains almost the same with 65 percent of planets having an absolute $|\Delta n|<0.1$. However, we also found that more than 60 per cent of hot Jupiters are clustered either at the orbital rank $n=0.5$ (which possibly represents the disc magnetospheric inner radius) or at $n=1$ (the corotation orbit), evidence of synchronization and spin-orbit coupling. Out of those, we found the majority of hot Jupiters with host-stars rotation of $P_{\text {rot }}<$ 10 days $\left(T_{\text {eff }}>6000 \mathrm{~K}\right)$ to be synchronized at the corotation orbit $n=1$, while the majority of hot Jupiters with star rotation of $P_{\text {rot }}>10$ days $\left(T_{\text {eff }}<6000 \mathrm{~K}\right)$ are orbiting even closer at $n=0.5$, i.e. at the proposed disc magnetospheric truncation radius.

We also examined the 49 multi-planetary systems in our samples, in which 6 systems harbor hot Jupiter planets, and found that the multi-planetary systems having hot Jupiters tend to have on average a lower absolute deviation from half-integer values (mean $|\Delta n|=0.086$ ) than multi-planetary systems that do not harbor any hot Jupiters (mean $|\Delta n|=0.107$ ). This implies that quantization features may be more pronounced in multi-planetary systems with hot Jupiters. However, the sample is still too small to draw any solid conclusions at this stage; this will have to await the discovery of more multi-planetary systems with hot Jupiters.

\subsection{The Role of Resonance Trapping in Forming Discrete Planetary Orbits}

Resonance mechanisms may play an important role in explaining the quantized planetary orbits. Mean-motion resonances, for instance, have already been used to explain the sequence of planetary spacing in the empirical Titius-Bode law (e.g. Patterson 1987). Additionally, mean-motion resonances were shown to influence the formation sites of protoplanets and were proposed as a means to halt planetary migration (resonance trapping). The migration of solid particles in a protoplanetary disc causes their orbits to decay and both eccentricity and inclination are damped with the loss of angular momentum. Under certain initial conditions, however, resonance between the planetesimals and an already formed planet embryo can counteract this orbital decay and trap the particles in a stable resonant orbit. There are strong indications that this mechanism can also explain the near-commensurabilities of the Solar System outer planets (Beauge et al. 1994; Malhotra 1995), as well as the spacing of the terrestrial planets (Laskar 1997).

Furthermore, resonance trapping was shown to be at work in more than 20 per cent of the 19 multiple exoplanetary systems considered by Tinney et al. (2006). Motivated by these observations, different studies have shown that during migration the capture of giant planets into resonances is a natural expectation (Nelson \& Papaloizou 2002). The planets subsequently migrate maintaining this commensurability. Resonance was also shown to play a key role in the formation of concentric density rings in the hybrid gravitational instability model of planet formation (Durisen et al. 2005). These resonant disc structures act as traps for infalling protoplanetary seeds and migrating planets and may provide a natural explanation for planetary orbits of higher discrete ranking $(n>1)$. Similarly, within the context of multiple planets forming in a disc, migration of the innermost planet might be stopped by either the magnetospheric gap at the truncation radius $(n=0.5)$ or by the star's tidal barrier at the corotation radius $(n=1.0)$. The size of these inner orbits is highly dependent on the stellar rotation rate. Now a second protoplanet approaching the star would stop when entering a low-order resonance with the innermost planet. The second planet's orbit would then be expected to correlate with the star's rotation rate as well. Therefore, the various features of resonance mechanisms may also provide a physical justification for the observed quantization in planetary orbits and their dependence on the star's rotation period. The multi-planetary system of G1876 provides evidence for this. The inner planet G1876d occupies the orbital rank of $n=0.6$, which we suggest may correspond to the disc magnetospheric truncation radius. The remaining planets, G1876 c, b, e, are locked in a 1:2:4 Laplace resonance (with orbital ranks $n=1.58$, 2.00, 2.54 respectively).

\section{Conclusion and Prospects}

We have shown that the orbital structure of planetary systems exhibits quantized features that must have evolved from the dynamical process of planetary formation. Our results demonstrate that planetary orbital periods and the parent star rotation period are correlated by discrete integer or half-integer values. Of course, the orbital period quantization also implies a quantization of planetary angular momenta, semi-major axes, and mean orbital velocities as well. This was confirmed for the Solar System and statistically verified over a list of 443 exoplanets, using both present rotation periods and those estimated at the fiducial formation age of $650 \mathrm{Myr}$. The statistical probability to obtain these results by pure chance is $p<0.046$. Future measurements for more accurate values of stellar rotation are needed to reduce uncertainties and support the conclusions presented here.

The quantization in planetary orbits is a function of stellar rotation and, consequently, is not related to any arbitrary universal constant (as in Nottale et al. 1996) but 
is specific to each exoplanetary system. Stellar rotation and the transfer of angular momentum play a key role in several planetary formation processes, such as tidal dissipation, disc truncation, and resonance, all of which could play a role in the resulting quantization reported here. Further investigation is required to understand the role of these physical processes.

The dependency on the central star's rotation rate corresponds to a strikingly similar relationship between the atomic orbital quantization (in integer multiples of the reduced Planck's constant $\hbar$ ) and the central proton's spin $(1 / 2 \hbar)$ in Bohr's model of the hydrogen atom. The quantization dependency on central body rotation, from atomic scales to large-scale gravitational systems, may be at the heart of a more general natural law or self-organizing principle that guides the formation of all rotating systems. Therefore, in addition to planetary systems, future investigation into the applications of this quantum-like model to other gravitational systems such as planetary satellites and ring systems, binary stars, and galactic centers, is intended to identify any discrete or quantized features in their orbital structure, and to validate the role and dependency of that quantization on the central body rotation rate. In concluding, the quantization of planetary orbits in half-integer multiples of the parentstar rotation period is now a statistically significant fact that should not be ignored in future models of planet formation.

\section{Acknowledgments}

We thank the anonymous referee for the valuable comments and for proposing the test in Section 5.5. This research has made use of the Exoplanet Orbit Database and the Exoplanet Data Explorer at http:// exoplanets.org, as well as the Extrasolar Planets Encyclopedia at http: / / exoplanet.eu/ by Jean Schneider.

\section{References}

Allende Prieto, C. \& Lambert, D. L., 1999, A\&A, 352, 555

Alonso, R. et al., 2004, ApJ, 613, L153

Alsubai, K. A. et al. 2010, MNRAS. Submitted; available from arXiv e-print: arXiv: $1012.3027 \mathrm{v} 2$.

Alves, S., do Nascimento Jr. J. D. \& de Medeiros J. R., 2010, MNRAS. Submitted; August 2010: available from arXiv e-print: arXiv: $1007.0145 \mathrm{v} 2$

Anderson, et al. PASP. Submitted, available from e-print arXiv: $1101.4643 \mathrm{v} 1$

Bakos, G. et al., 2009, ApJ, 707, 446

Bakos, G. et al., 2010, ApJ. Submitted; August 2010: available from arXiv e-print: arXiv:1008.3388v1.

Bakos, G. et al., 2007, ApJ, August 2010: Available from arXiv e-print: arXiv:0710.1841v1.

Baliunas, S., Sokoloff, D. \& Soon, W., 1996, ApJ, 457, L99

Barge, P. et al., 2008, A\&A, 482, 17

Beauge, C., Aarseth, S. J. \& Ferraz-Mello, S., 1994, MNRAS, 270,21

Boisses, et al., 2010, A\&A, Accepted. August 2010: available from arXiv e-print: arXiv:1006.4984v1.

Bonfils, X. et al., 2010, A\&A, 2007 Submitted; August 2010: available from arXiv e-print: arXiv0704.0270B.
Bonomo, et al., 2010, A\&A, accepted; August 2010: available from arXiv e-print: arXiv:1006.2949.

Borde, et al., 2010, A\&A, accepted; August 2010: available from arXiv e-print: arXiv: 1008.0325.

Boss, A. P., 2005, Evolution of the Solar Nebula. VII. Formation and Survival of Protoplanets Formed by Disk Instability. ApJ, 629,535

Bouchy, et al., 2009, A\&A, 496, 527

Bouchy, et al., 2010, A\&A, Accepted; August 2010: available from arXiv e-print: arXiv: $1006.2605 \mathrm{v} 1$.

Buchhave, et al., 2010, ApJ, 720, 1118

Butler, P. et al., 2006, ApJ, 646, 505

Cabrera, et al., A\&A, Accepted; August 2010: available from arXiv e-print: arXiv:1007.5481.

Cameron, et al., 2010, MNRAS, Submitted; August 2010: available from arXiv e-print: arXiv:1004.4551.

Chang, H.-Y., 2010, Journal of Astronomy and Space Sciences, 27, 1

Cox, A., 1999, Allen's Astrophysical Quantities, 4th Ed., SpringerVerlag, New York.

D’Angelo, G., Durisen, R. \& Lissauer, J., 2010 Submitted, August 2010: available from arXiv e-print: arXiv:1006.5486v1.

Da Silva, R. et al., 2007, A\&A, 473, 323

Duncan, D., Baliunas, S., Noyes, R., Vaughan, A., Frazer, J. \& Lanning, H., 1984, PASP, 96, 707

Durisen, R., Cai, K., Mejía, A. \& Pickett, M., 2005, Icarus, 173, 417

Eggenberger, A., Mayor, M., Naef, D., Pepe, F., Queloz, D., Santos, N. C., Udry, S. \& Lovis, C., 2006, A\&A, 447, 1159

Enoch, et al., 2010, MNRAS, Submitted

European Space Agency (ESA)1997, The Hipparcos \& Tycho Catalogue, ESA SP, 1200. VizieR On-line Data Catalog: I/239

Gandolfi, et al., 2010, A\&A, Submitted; July 2010; available from arXiv e-print: arXiv: $1009.2597 \mathrm{v} 1$

Glebocki, R. \& Gnacinski, P., 2005, The Catalogue of Rotational Velocities of Stars, ESA SP, 560, 571

Gonzalez, G., Carlson, M. K. \& Tobin, R. W., 2010, MNRAS, 403, 1368

Griv, E. \& Gedalin M., 2005, Formation of the solar system by instability. DPPS, \#197, Belgrade, Ed. Knezevic Z., Milani A., Cambridge Univ. Press, p.97-106.

Guenther, et al., 2009, A\&A, 507, 1659

Haghighipour, N. \& Boss, A. P., 2003, ApJ, 598, 1301

Haghighipour, N. et al., 2010, ApJ, 715, 271

Han, I. et al., 2010, A\&A, 509, A24

Hartman, J. et al., 2010, ApJ, Submitted; August 2010: available from arXiv e-print: arXiv:1007.4850v1.

Hébrard, G. et al., 2010a, A\&A, 512, A46

Hébrard, G. et al., 2010b, A\&A, 513, A69

Hekker, S. \& Melendez, J., 2007, A\&A, 475, 1003

Hellier, et al., 2010, ApJL, Submitted; August 2010: available from arXiv e-print: arXiv: $1009.5318 \mathrm{v} 1$

Henry, G. W., Baliunas, S. L., Donahue, R. A., Fekel, F. C. \& Soon, W., 2000, ApJ, 531, 415

Howard, A. W. et al., 2010, ApJ, 721, 1467

Howard, A. W. et al., 2010a, ApJ, Submitted; August 2010: available from arXiv e-print: arXiv:1008.3898v1.

IAU Minor Planet Center website, August 2010 b; available from http: //www. cfa.harvard.edu/iau/Ephemerides / Comets

Jenkins, J. S., Ramsey, L. W., Jones, H. R. A., Pavlenko, Y., Gallardo, J., Barnes, J. R. \& Pinfield, D., 2009, AJ, 704, 975

Johns-Krull, C. et al., 2008, AJ, 677, 657

Johnson, J. A. et al., 2010, PASP, 122, 701

Kharchenko, N. V., 2001, Kinematics and Physics of Celestial Bodies, 17, 409

Kipping, D. M. et al., 2010, AJ, Submitted; August 2010: available from arXiv e-print: arXiv: $1008.3389 \mathrm{v} 1$.

Kovács, G. et al., 2007, ApJ, 670, L41

Kovács, G. et al., 2010, ApJ, Submitted; August 2010: available from arXiv e-print: arXiv:1005.5300v1. 
Lanza, A. et al., 2010, A\&A, Accepted. Available from arXiv e-print: arXiv:1005.3602v1.

Laskar, J., 1997, A\&A, 317, L75

Lin, D. N. C. \& Papaloizou, J. C. B., 1996, A\&A, 34, 703

Lovis, C. et al., 2010, A\&A, Submitted; Lowell observatory webpage, August 2010: available from: ftp: / / ftp. lowell. edu/pub/elgb/astorb.html

Malhotra, R., 1995, AJ, 110, 420

Mandushev, G. et al., 2007, ApJL, 667, L195

Masana, E., Jordi, C. \& Ribas, I., 2006, A\&A, 450, 735

Masset, F., Morbidelli, A., Crida, A. \& Ferreira, J., 2006, ApJ, 642, 478

Mayor, M., Udry, S., Naef, D., Pepe, F., Queloz, D., Santos, N. C. \& Burnet, M., 2004, A\&A, 415, 391

Mayor, M. et al., 2009, A\&A, 493, 639

McCullough, P. et al., 2006, AJ, 648, 1228

Melo, C. et al., 2006, A\&A, 460, 251

Mordasini, C. et al., 2010, A\&A

Moutou, C. et al., 2009, A\&A, 496, 513

Naef, D. et al., 2010, A\&A, Accepted; available from e-print arXiv:1008.4600v1.

Nelson, R. \& Papaloizou, J. C. B., 2002, MNRAS, 333, L26

Niedzielski, A. et al., 2007, ApJ, Submitted; August 2010: available from arXiv e-print: arXiv:0705.0935v1.

Niedzielski, A. et al., 2009a, ApJ, 693, 276

Niedzielski, A., Nowak, G., Adamow, M. \& Wolszczan, A., 2009b, AJ, 707, 768

Nordström, B. et al., 2004, A\&A, 418, 989

Nottale, L., 1996, A\&A, 315, L09

Nottale, L., 1997b, A\&A, 327, 867

Nottale, L., Schumacher, G. \& Gay, J., 1997a, A\&A, 322, 1018

Nottale, L., Ceccolini, D., Da Rocha, D., Tran-Minh, N., Galopeau, P. \& Schumacher, G., 2004, ASP Conf. Ser.: Extrasolar Planets: Today and Tomorrow, 321, 355

O’Donovan, F. et al., 2007, ApJ, 663, L37

Pál, A. et al., 2008, ApJ, 680, 1450

Pasinetti Fracassini, L. E., Pastori, L., Covino, S. \& Pozzi, A., 2001, A\&A, 367, 521

Patel, S. et al., 2007, ApJ, 665, 744

Patterson, C. W., 1987, Icarus, 70, 319

Poveda, A. \& Lara, P. The exoplanetary system of 55 Cancri and the Titiu-Bode law, 2008, arXiv: 0803.2240.
Queloz, D. et al., 2010, A\&A, 517, L1

Radick, R., Thompson, D., Lockwood, G., Duncan, D. \& Baggett, W., 1987, AJ, 321, 459

Radick, R., Lockwood, G., Skiff, B. \& Thompson, D., 1995, ApJ, 452, 332

Rasio, F. A., Tout, C. A., Lubow, S. H. \& Livio, M., 1996, AJ, 470, 1187

Rivera, E. et al., 2010, ApJ, 708, 1492

Rubcic, A. \& Rubcic, J., 1998, Fizika B, 7, 1

Rubcic, A. \& Rubcic, J., 1999, Fizika B, 2, 45

Rutten, R, 1987, A\&A, 177, 131

Santos, N. C. et al., 2002, A\&A, 392, 215

Scholz, A. \& Eisloffel, J., 2007, MNRAS, 381, 1638

Schroeder, C., Reiners, A. \& Schmitt, J., 2009, A\&A, 493, 1099

Schuler, S. et al., 2005, ApJ, 632, L131

Simpson, E. K. et al., 2010, AJ, Submitted; August 2010: available from arXiv e-print: arXiv:1008.3096v1.

Skumanich, A., 1972, AJ, 171, 565

Snellen, I. et al., 2009, A\&A, 497, 545

Soderblom, D. R., Jones, B. \& Fischer, D., 2001, AJ, 563, 334

Street, R. et al., 2010, ApJ, 720, 337

Tingley, B. et al. 2010, A\&A, Accepted; available from e-print: arXiv:1101.1899v1.

Tinney, C., Butler, P., Marcy, G., Jones, H., Laughlin, G., Carter, B., Bailey, J. \& O’Toole, S., 2006, ApJ, 647, 594

Tokovinin, A. \& Smekhov, M. G., 2002, A\&A, 382, 118

Torres, G., Quast, G. R., da Silva, L., de la Reza, R., Melo, C. \& Sterzik, M., 2006, A\&A, 460, 695

Torres, G. et al., 2010, ApJ, 715, 458

Trilling, D., Lunine, J. \& Benz, W., 2002, A\&A, 394, 241

Vogt, S. et al., 2010, ApJ, 708, 1366

Watson, C., Littlefair, S., Cameron, A., Dhillon, V. \& Simpson, E., 2010, MNRAS, Submitted; Available from arXiv e-print: arXiv:1006.2069v1.

West, R. et al., 2010, AJ, http: / /www. superwasp.org/ documents/west2010_wasp28.pdf

Wright, J. \& Marcy, G. Exoplanet Orbit Database. August 2010: available from Exoplanet Data Explorer Table http:// exoplanets.org/

Yi, I., 1995, ApJ, 442, 768

Zacharias, N., Monet, D., Levine, S. E., Urban, S., Gaume, R. \& Wycoff, G., 2005, AAS, 205, 48.15 